\title{
Poly(thioether)s from Closed-System One-Pot Reaction of Carbonyl Sulfide and Epoxides by Organic Bases
}

Cheng-Jian Zhang ${ }^{1}$, Tian-Cheng Zhu², Xiao-Han Cao ${ }^{1}$, Xin Hong ${ }^{2}$, Xing-Hong Zhang*1

1 MOE Key Laboratory of Macromolecular Synthesis and Functionalization, Department of Polymer Science and Engineering, Zhejiang University, Hangzhou 310027, China.

${ }^{2}$ Department of Chemistry, Zhejiang University, Hangzhou 310027, China 


\section{Table of Contents}

Table S1. ROP of the purified mixture of 1 and 2 at $60{ }^{\circ} \mathrm{C}$ using organic base combined with benzyl alcohol $(\mathrm{BnOH})$

Figure S1. ${ }^{1} \mathrm{H}$ NMR of PO/COS/DBU of $1 / 1.2 / 0.01$ at $80{ }^{\circ} \mathrm{C}$ for $48 \mathrm{~h}$ in a $10 \mathrm{ml}$ autoclave . .5

Scheme S1. Mechanism of the cyclization of PO with COS using DBU...............................................................6

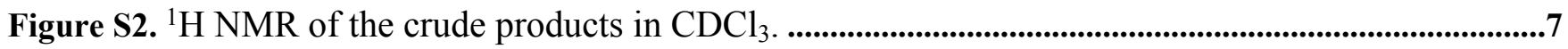

Figure S3. ${ }^{1} \mathrm{H}$ NMR of the crude products generated from the ROP of 1 and 2 at $120{ }^{\circ} \mathrm{C}$ for 5 min and 10 min in $\mathrm{CDCl}_{3}$.

Figure S4. MALDI-TOF MS spectrum of the PPS oligomers. Poly $(\mathrm{PS})_{7}=\left[\mathrm{DBU}+\left(\mathrm{C}_{2} \mathrm{H}_{6} \mathrm{~S}\right)_{7}+\mathrm{H}+\right.$ Maleimide $+\mathrm{K}^{+}, \mathrm{m} / \mathrm{z}$ 808.42] or $\left[\mathrm{C}_{2} \mathrm{H}_{5} \mathrm{O}+\left(\mathrm{C}_{2} \mathrm{H}_{6} \mathrm{~S}\right)_{7}+\mathrm{H}+\right.$ Maleimide $+\mathrm{K}^{+}, \mathrm{m} / \mathrm{z}$ 701.25]. .

Scheme S2. Possible pathways of the chain initiation of ROP of cyclic thiocarbonate 1 by DBU. . .11

Figure S5. Free energies of pathways in Scheme S2 ..........................................................................................12

Figure S6. Free energies of two pathways of the ring-opening reaction . .13

Scheme S3. Other possible pathways of the chain growth of ROP of cyclic thiocarbonate 1 . . .14

Figure S7. Free energies in Scheme S3 . .15

Table S2. Energies, enthalpies, and free energy of the calculated structures .18

Cartesian Coordinates for the Optimized Structures . .19

Figure S8. ${ }^{1} \mathrm{H}$ NMR of the crude products varied with time at $80{ }^{\circ} \mathrm{C}$ with a) $\mathrm{PO} / \mathrm{COS} / \mathrm{DBU} / \mathrm{TU}$ $=100 / 1.2 / 1 / 2$, b) $\mathrm{PO} / \mathrm{COS} / \mathrm{DBU}=100 / 1.2 / 1.0$.

Figure S9. The content of multiple components varied with time at $80{ }^{\circ} \mathrm{C}$ with a) DBU/TU, b) single DBU . .37

Figure S10. ${ }^{1} \mathrm{H}$ and ${ }^{13} \mathrm{C}$ NMR of the generated poly(thioether) from epoxide 2, Table 3.............................38

Figure S11. ${ }^{1} \mathrm{H}$ and ${ }^{13} \mathrm{C}$ NMR of the generated poly(thioether) from epoxide 3, Table 3............................39

Figure S12. ${ }^{1} \mathrm{H}$ and ${ }^{13} \mathrm{C}$ NMR of the generated poly(thioether) from epoxide 4, Table 3............................40 
Figure S13. ${ }^{1} \mathrm{H}$ and ${ }^{13} \mathrm{C}$ NMR of the generated poly(thioether) from epoxide 8, Table 3.

Figure S14. ${ }^{1} \mathrm{H}$ and ${ }^{13} \mathrm{C}$ NMR of the generated poly(thioether) from epoxide 9, Table 3.............................42

Figure S15. ${ }^{1} \mathrm{H}$ and ${ }^{13} \mathrm{C}$ NMR of the generated poly(thioether) from epoxide 10, Table 3...........................43

Figure S16. ${ }^{1} \mathrm{H}$ and ${ }^{13} \mathrm{C}$ NMR of the generated poly(thioether) from epoxide 11, Table 3..........................44

Figure S17. ${ }^{1} \mathrm{H}$ and ${ }^{13} \mathrm{C}$ NMR of the generated poly(thioether) from epoxide 12, Table 3...........................45

Figure S18. DSC curves of the generated poly(thioether)s from a) epoxides 1-4, b) epoxide 8, c)

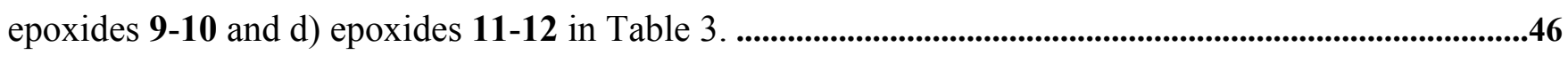

Figure S19. GPC curves of the generated poly(thioether)s from a) epoxides 1-4, b) epoxides 5-8, c) epoxides 9-12 in Table 3.....................................................................................................................................47

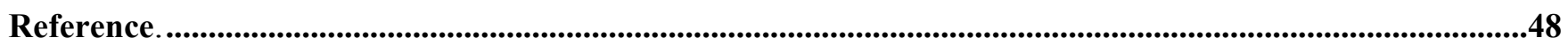


Table S1. ROP of the purified mixture of 1 and 2 at $60^{\circ} \mathrm{C}$ using organic base combined with benzyl alcohol $(\mathrm{BnOH})^{\text {a }}$

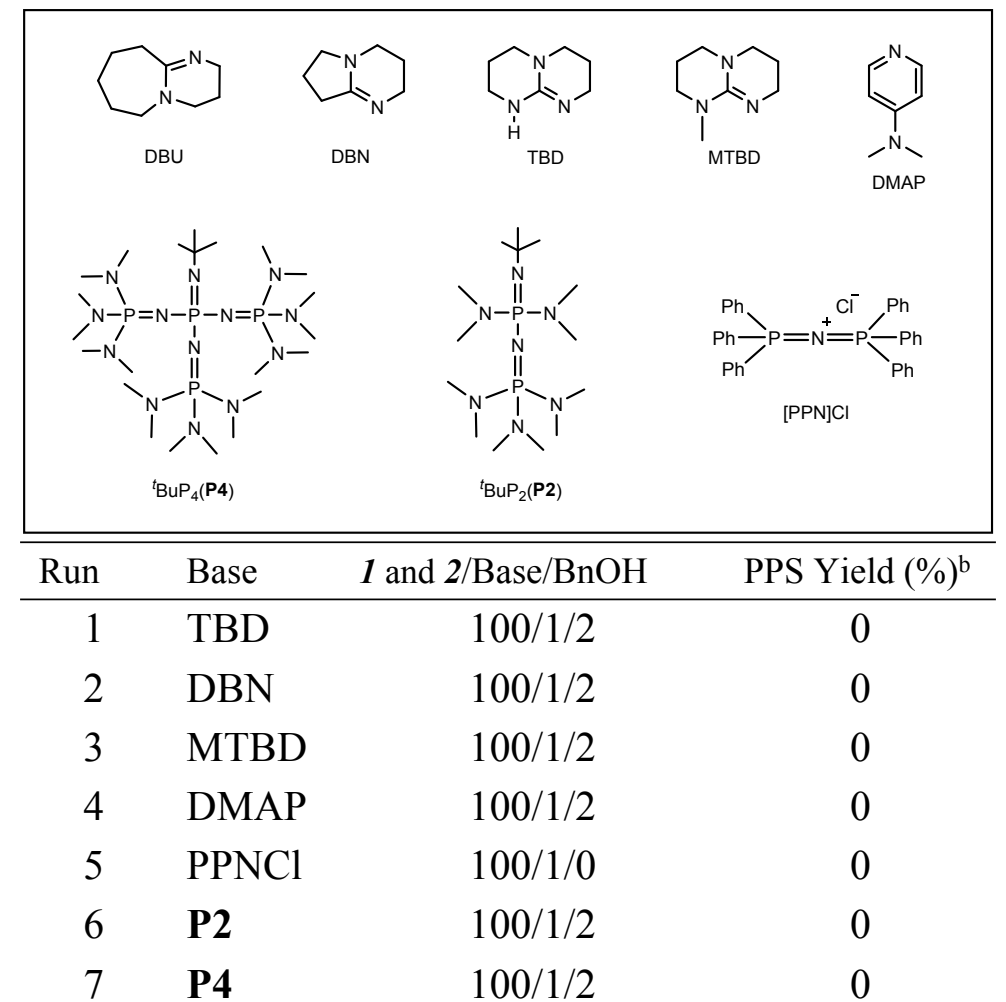

${ }^{\text {a }}$ Reactions were run at $60^{\circ} \mathrm{C}$ for $72 \mathrm{~h}$, in neat purified mixture of 1 and $2(1.0 \mathrm{ml})$ in a $10 \mathrm{ml}$ autoclave. ${ }^{b}$ Polymer yield was determined by ${ }^{1} \mathrm{H}$ NMR spectroscopy. 

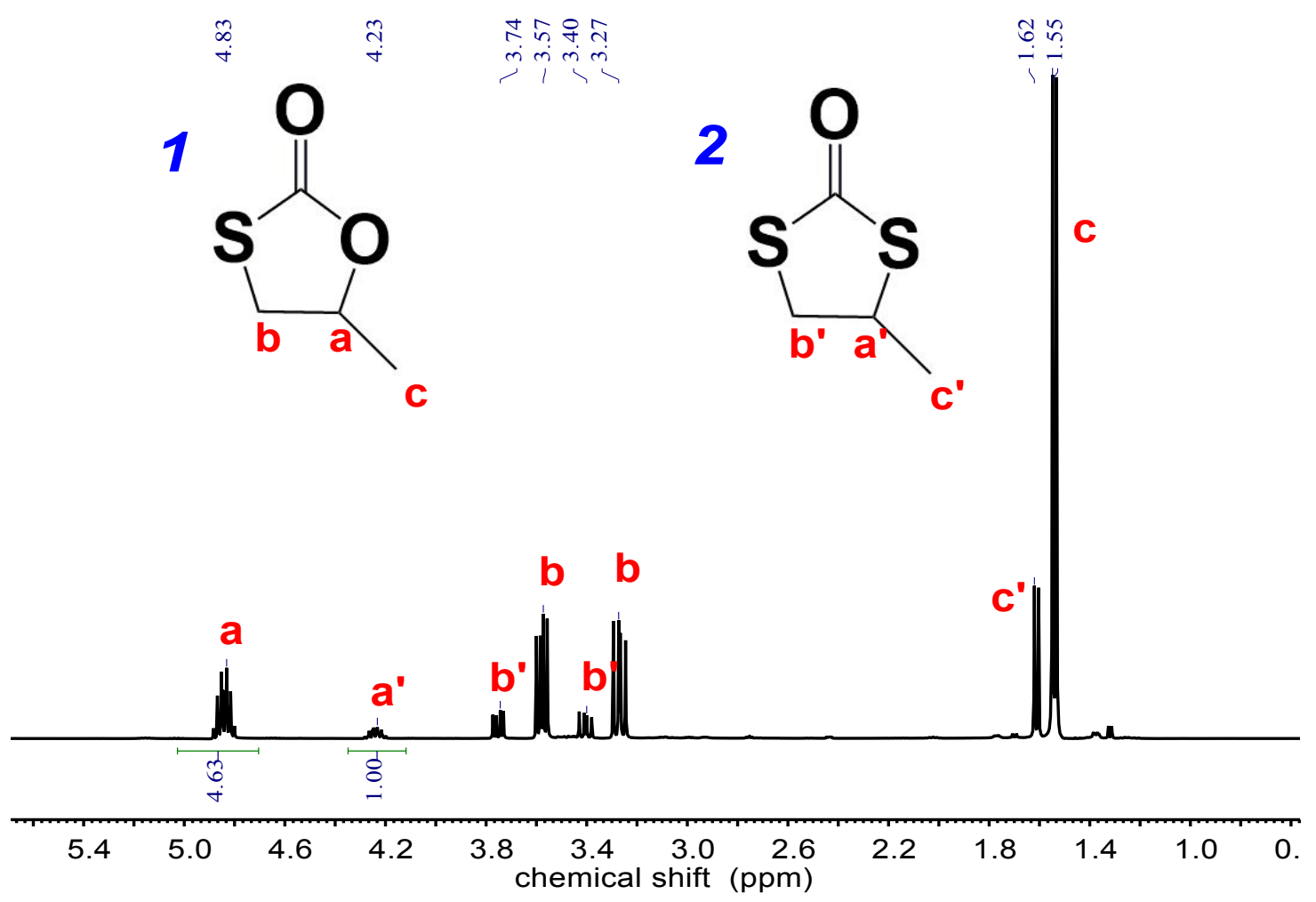

Figure S1. ${ }^{1} \mathrm{H}$ NMR of $\mathrm{PO} / \mathrm{COS} / \mathrm{DBU}$ of $1 / 1.2 / 0.01$ at $80^{\circ} \mathrm{C}$ for $48 \mathrm{~h}$ in a $10 \mathrm{ml}$ autoclave. 
a

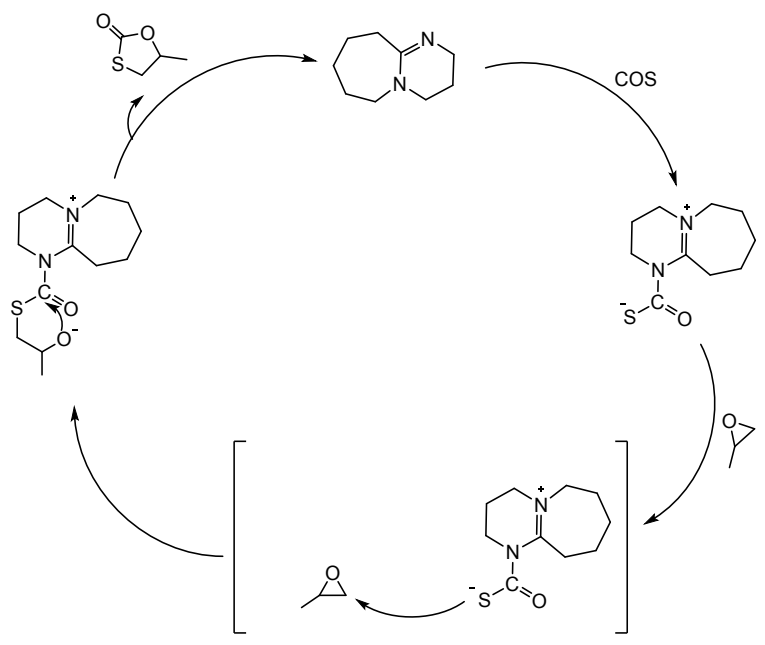

b

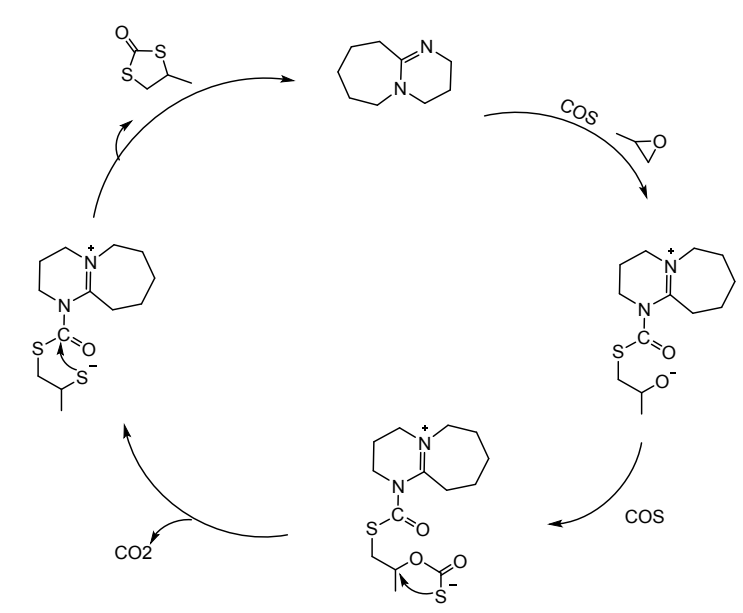

Scheme S1. The proposed zwitterionic process of the cyclization of PO with COS using DBU. 


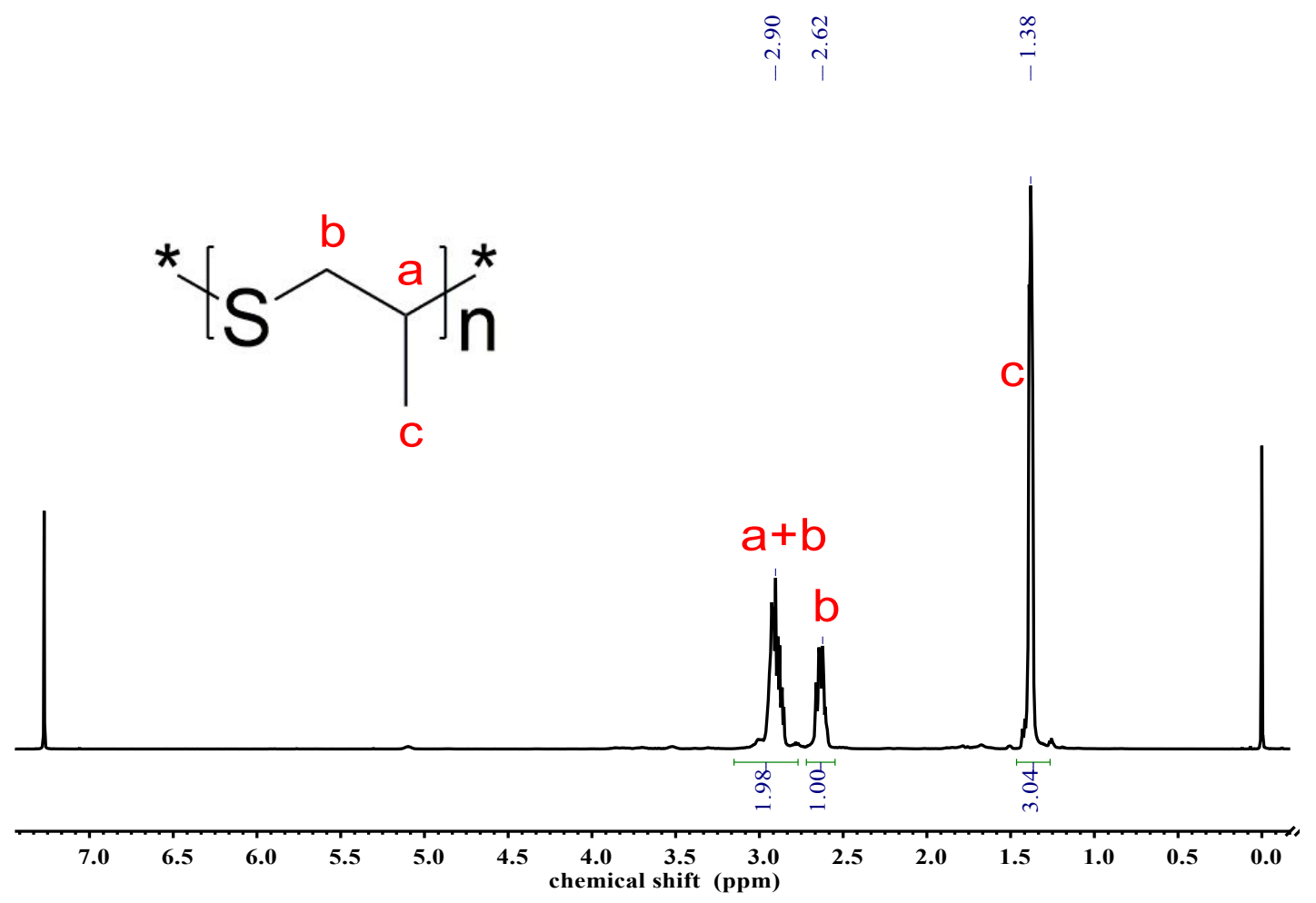

Figure S2. ${ }^{1} \mathrm{H}$ NMR of the crude products in $\mathrm{CDCl}_{3}$. 


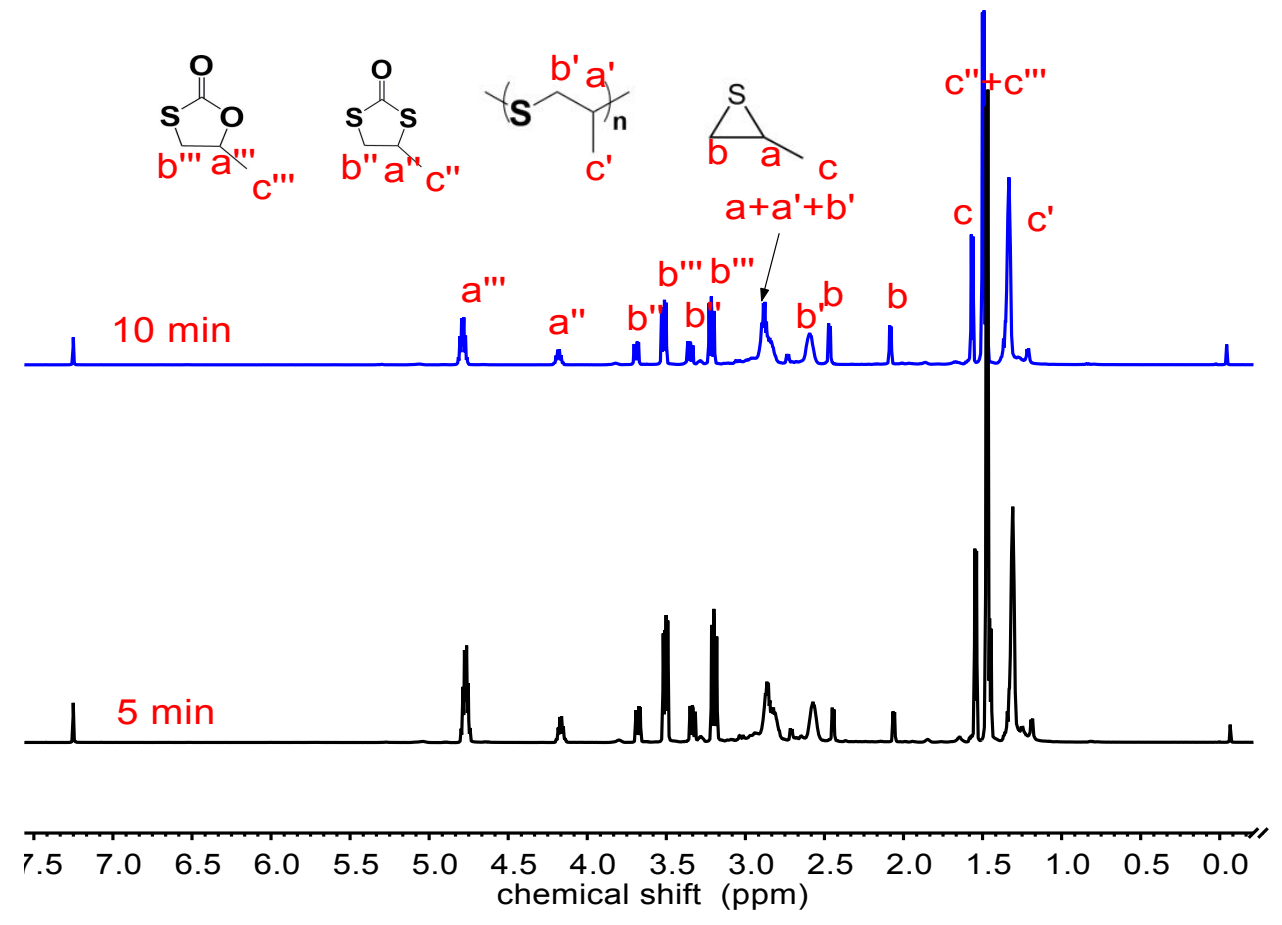

Figure S3. ${ }^{1} \mathrm{H}$ NMR of the crude products generated from the ROP of $\boldsymbol{1}$ and $\boldsymbol{2}$ at 120 ${ }^{\circ} \mathrm{C}$ for $5 \mathrm{~min}$ and $10 \mathrm{~min}$ in $\mathrm{CDCl}_{3}$. 


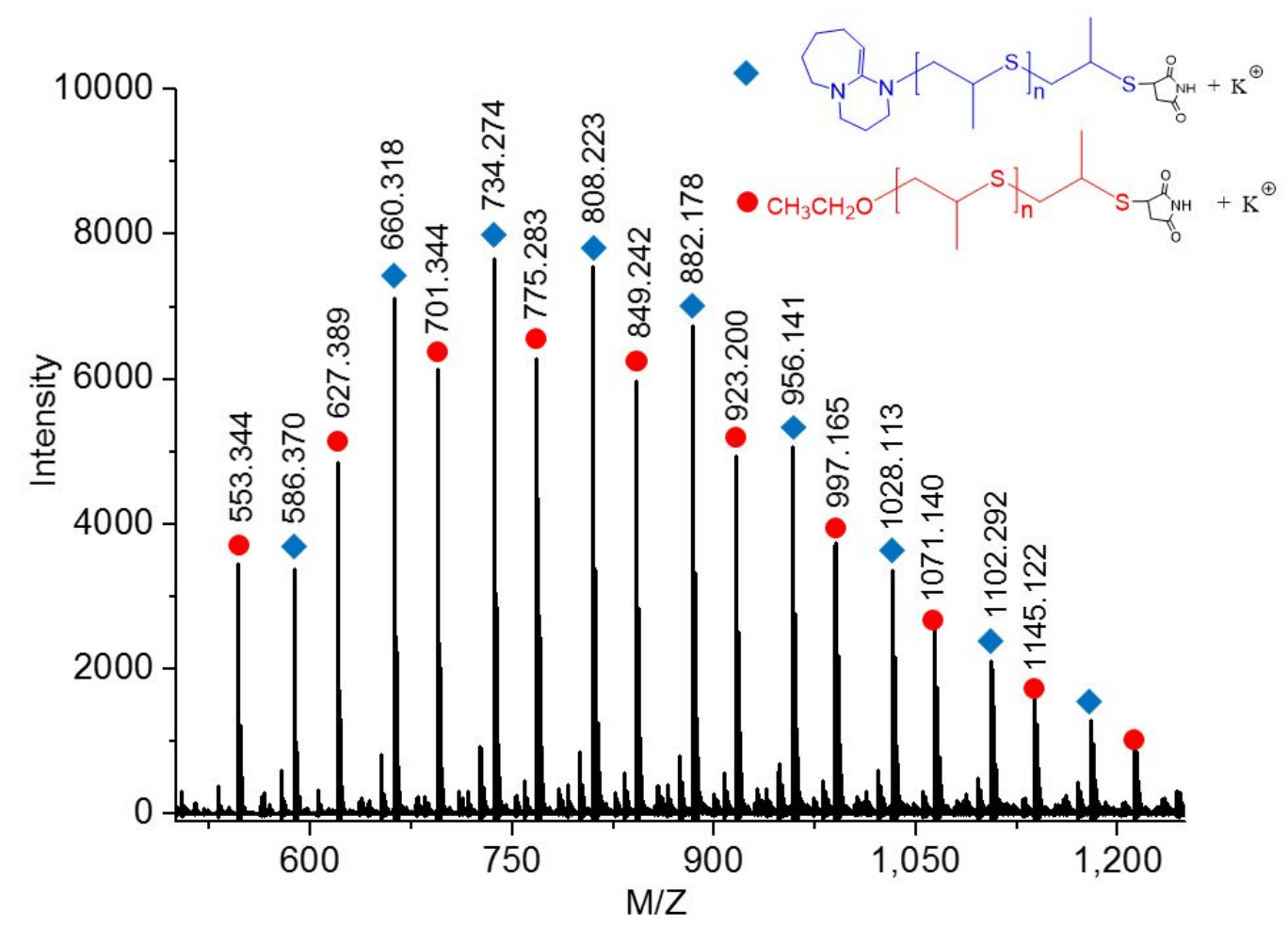

Figure S4. MALDI-TOF MS spectrum of PPS oligomers. Poly $(\mathrm{PS})_{7}=$ $\left[\mathrm{DBU}+\left(\mathrm{C}_{2} \mathrm{H}_{6} \mathrm{~S}\right)_{7} \quad+\mathrm{H}+\right.$ Maleimide $+\mathrm{K}^{+}, \quad \mathrm{m} / \mathrm{z} \quad$ 808.42] $\quad$ or $\quad\left[\mathrm{C}_{2} \mathrm{H}_{5} \mathrm{O}+\left(\mathrm{C}_{2} \mathrm{H}_{6} \mathrm{~S}\right)_{7}\right.$ $+\mathrm{H}+$ Maleimide $+\mathrm{K}^{+}, \mathrm{m} / \mathrm{z}$ 701.25]. 


\section{Computational details}

All DFT calculations were conducted with the Gaussian 16 software package ${ }^{1}$. Geometry optimizations of all the minima and transition states were performed using $\mathrm{B} 3 \mathrm{LY} \mathrm{P}^{2-3}$ functional with 6-31G(d) basis set, including Grimme's dispersion corrections ${ }^{4}$. Vibrational frequencies were computed at the same level to evaluate its zero-point vibrational energy (ZPVE) and thermal corrections at $298 \mathrm{~K}$ as well as to confirm whether each optimized stationary point is an energy minimum or a transition state. The solvation energies were evaluated by a self-consistent reaction field (SCRF) using M06-2x functional with $6-311+\mathrm{G}(\mathrm{d}, \mathrm{p})$ basis set and the PCM model ${ }^{5-7}$. In addition, to correct the Gibbs free energies under pressure of $1 \mathrm{~atm}$ to the standard state in solution $(1 \mathrm{~mol} / \mathrm{L})$, a correction of $\mathrm{RT} \ln \left(\mathrm{c}_{\mathrm{s}} / \mathrm{c}_{\mathrm{g}}\right)$ (about $1.9 \mathrm{kcal} / \mathrm{mol}$ ) is added to energies of all species except for gas. $\mathrm{c}_{\mathrm{s}}$ is the standard molar concentration in solution $(1 \mathrm{~mol} / \mathrm{L}), \mathrm{c}_{\mathrm{g}}$ is the standard molar concentration in gas phase $(0.0446 \mathrm{~mol} / \mathrm{L})$, and $\mathrm{R}$ is the gas constant. Extensive conformational searches for the intermediates and transition states have been conducted to ensure that the lowest energy conformers were located. The 3D diagrams of molecules were generated using CYLView ${ }^{8}$. 


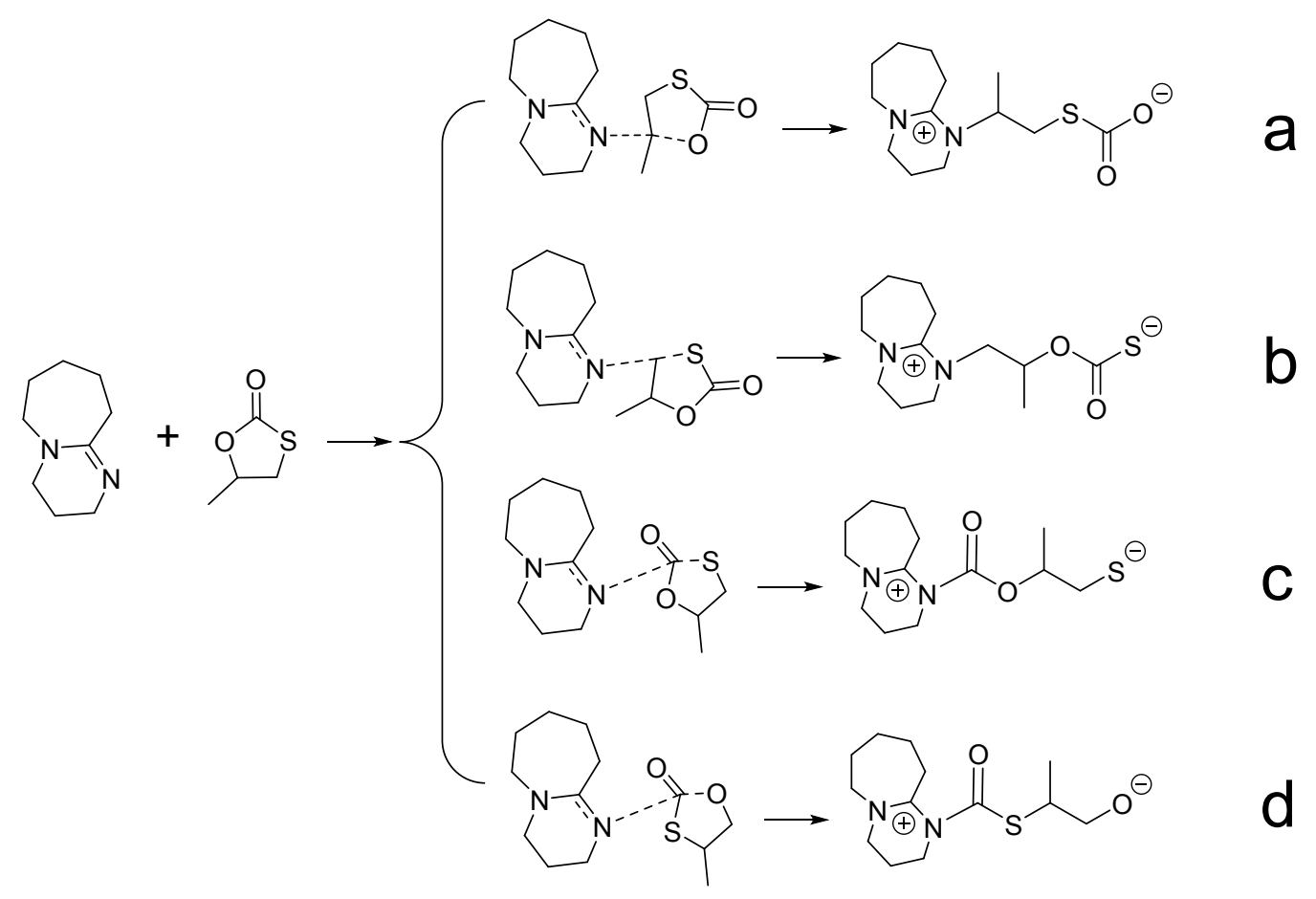

Scheme S2. Possible pathways of the chain initiation of ROP of cyclic thiocarbonate 1 by DBU. 

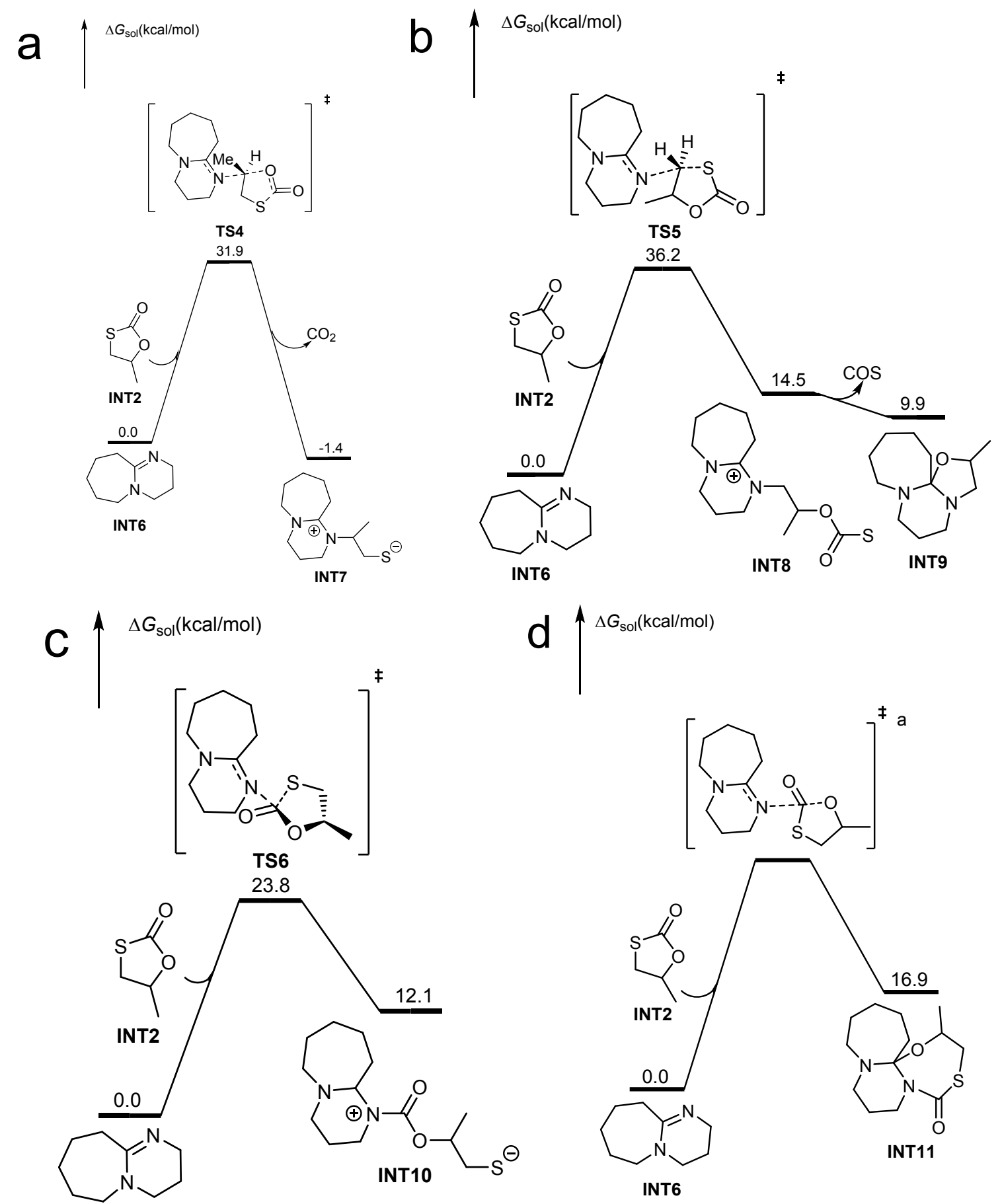

d $\uparrow^{\Delta G_{\mathrm{sol}}(\mathrm{kcal} / \mathrm{mol})}$

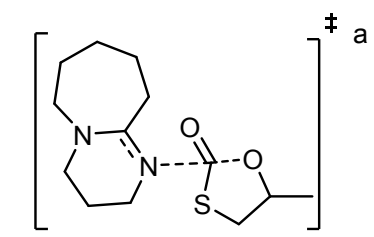

INT6

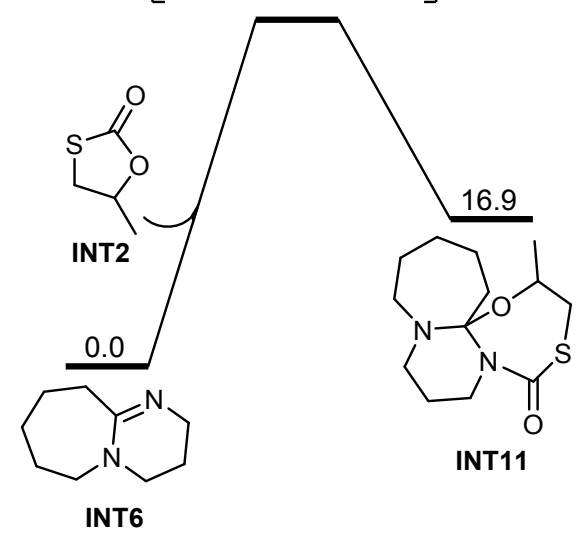

a:The transtion state cannot be located.

Figure S5. Free energies of the pathways in Scheme S2. Although TS6 has the lowest energy among the transition states, the thermodynamics of the pathway is not favorable. Therefore, we think pathway a is the most favorable one. 


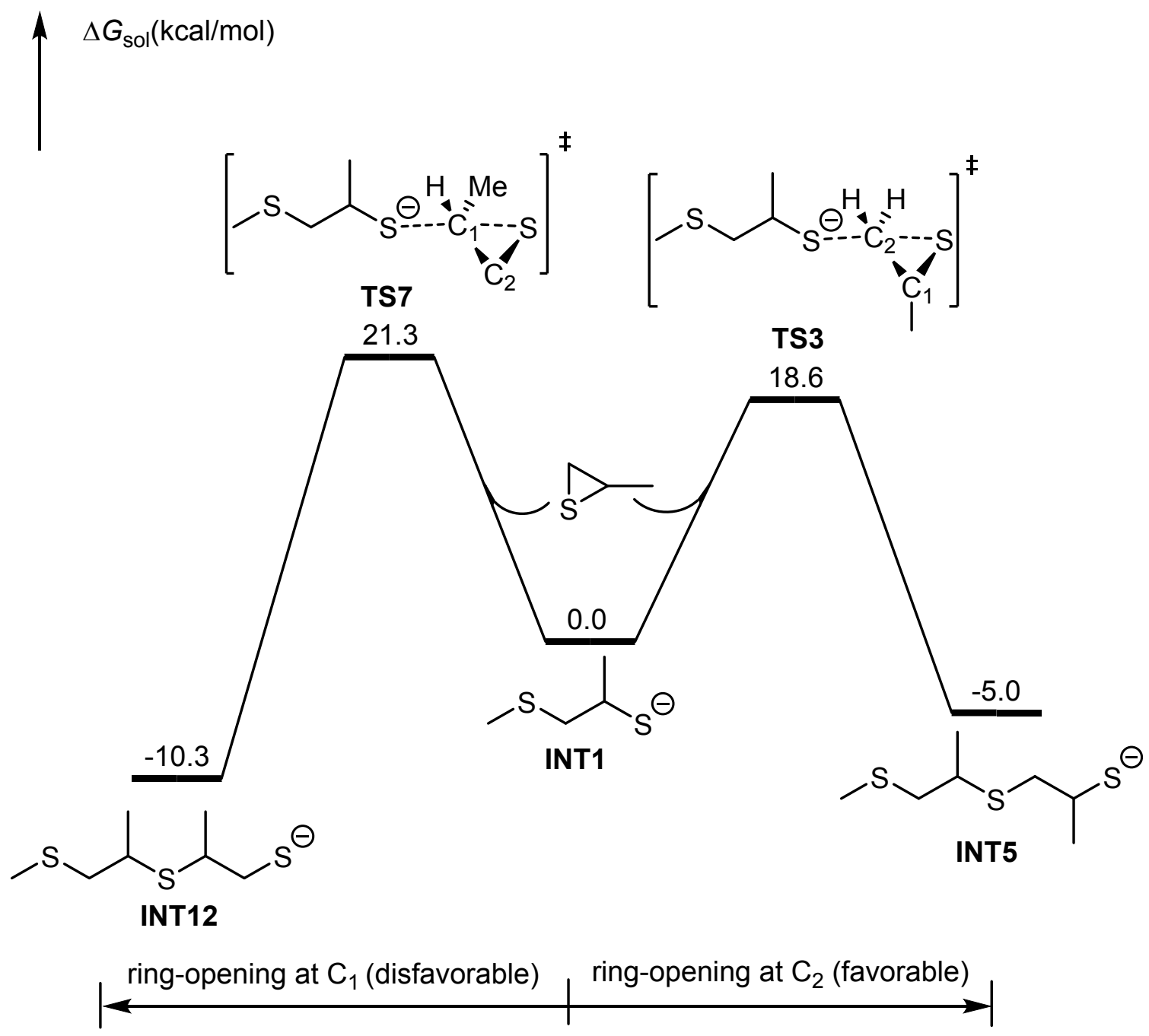

Figure S6. Free energies of two pathways of the ring-opening reaction. The right one is more favorable. 


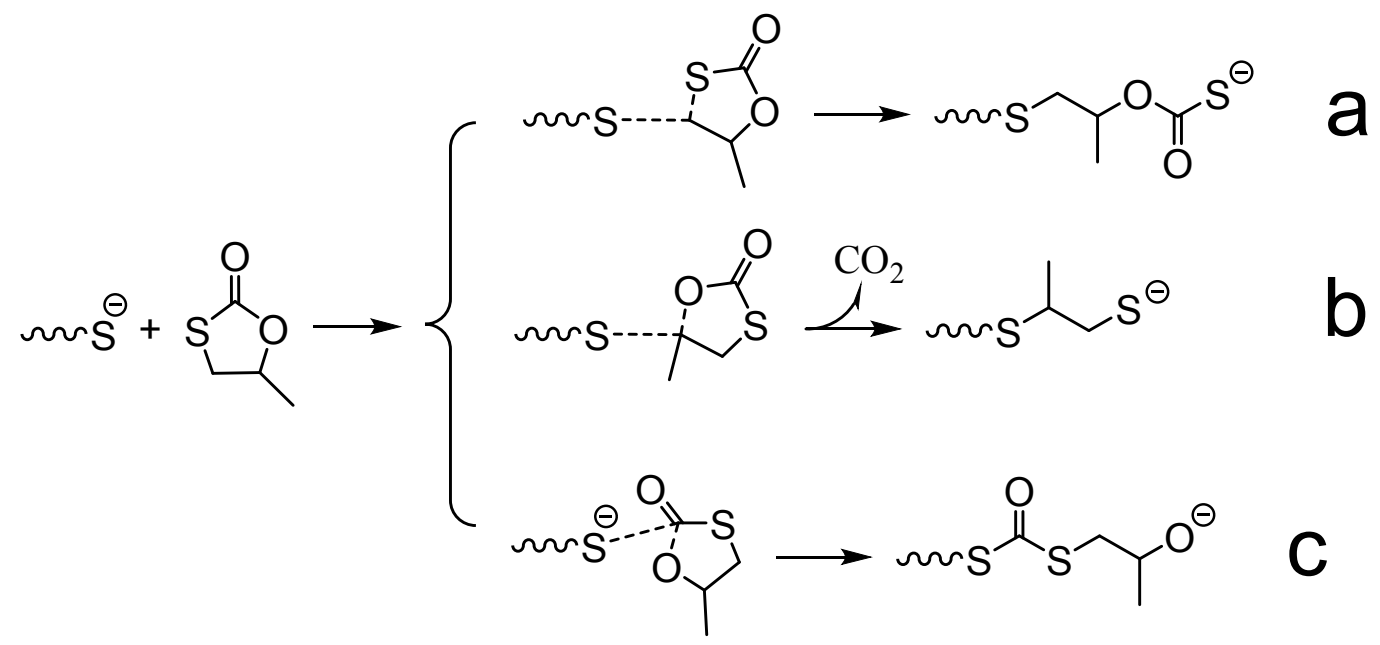

Scheme S3. Other possible reaction pathways of the chain growth of ROP of cyclic thiocarbonate 1 . 


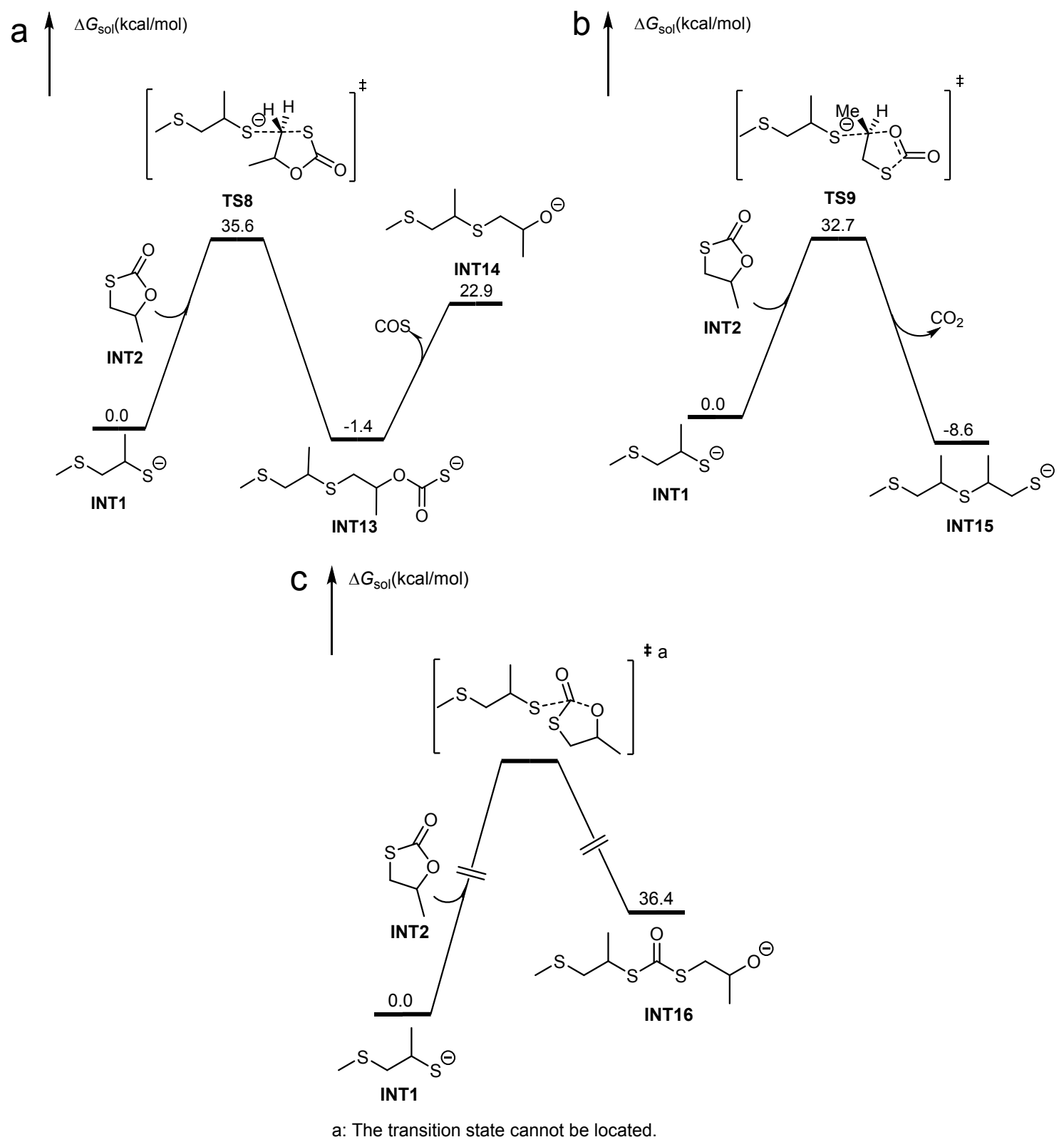

Figure S7. Free energies in Scheme S3. 
Table S2. Energies, enthalpies, and free energy of the calculated structures

Zero-point correction (ZPE), thermal correction to enthalpy $(\Delta \mathrm{H})$, thermal correction to Gibbs free energy $(\Delta \mathrm{G})$, energies $(E)$, enthalpies $(H)$, Gibbs free energies $(\mathrm{G})$ (in Hartree) and imaginary frequencies of the transition states were calculated at the M06-2x/6-311+G(d,p)-PCM(4-methyl-1,3-dioxolan-2-one)//B3LYP-D3(BJ)/6-31G(d) level of theory.

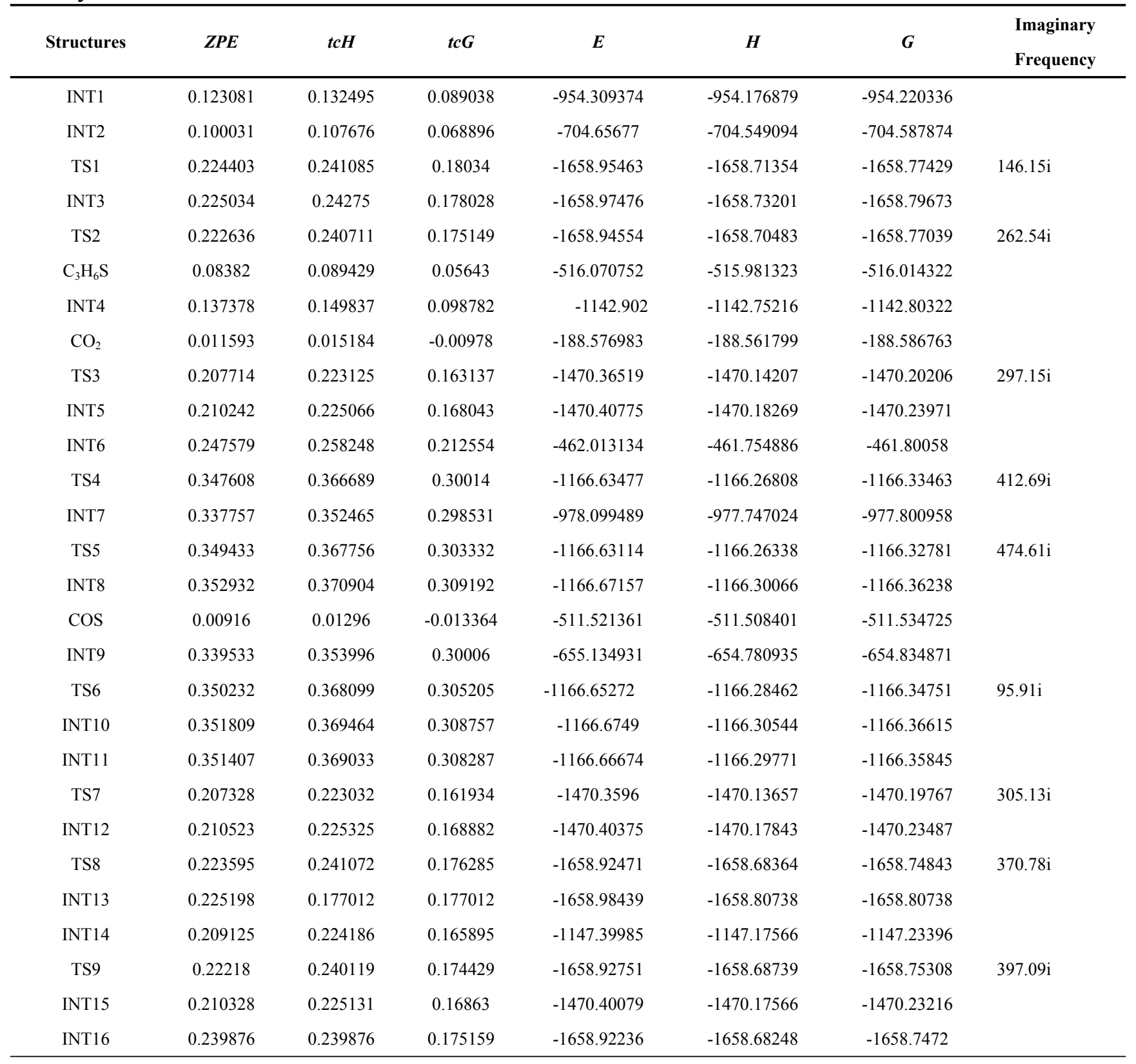




\section{Cartesian Coordinates for the Optimized Structures}

INT1

$\begin{array}{lrrc}\mathrm{C} & -2.97746400 & -0.81452100 & 0.25378300 \\ \mathrm{H} & -3.99694200 & -0.45119000 & 0.08838800 \\ \mathrm{H} & -2.86042700 & -1.05812100 & 1.31603200 \\ \mathrm{H} & -2.83047300 & -1.72913800 & -0.33218000 \\ \mathrm{~S} & -1.80435600 & 0.48879200 & -0.25746200 \\ \mathrm{C} & -0.20375700 & -0.43426100 & 0.12050000 \\ \mathrm{H} & -0.27812100 & -1.40339100 & -0.37858500 \\ \mathrm{C} & 1.05783800 & 0.28969900 & -0.34453900 \\ \mathrm{H} & 0.96374500 & 0.45803000 & -1.42854200 \\ \mathrm{~S} & 2.48444100 & -0.82537900 & -0.00560300 \\ \mathrm{H} & -0.16305700 & -0.59810700 & 1.20224200 \\ \mathrm{C} & 1.20783800 & 1.65554100 & 0.33601400 \\ \mathrm{H} & 0.36472200 & 2.33397700 & 0.12178800 \\ \mathrm{H} & 1.27748800 & 1.52180200 & 1.42261000 \\ \mathrm{H} & 2.13496400 & 2.13277600 & 0.00274200\end{array}$

INT2

$\mathrm{C}$

C

$\mathrm{C}$

$\mathrm{S}$

$\mathrm{H}$

$\mathrm{O}$

O

$\mathrm{C}$

$\mathrm{H}$

$\mathrm{H}$

$\mathrm{H}$

$\mathrm{H}$

$\mathrm{H}$

$$
0.67321700
$$

1.25849100

0.15946100

1.19757100

$-0.07276800$

$-0.38944400$

$-1.04521100$

$-0.67527800$

0.01928600

$-1.14020800$

1.13392800

$-0.02738400$

0.93146300

1.38596800

1.21500800

0.24391500

$-1.09786100$

$-0.03303900$

$-1.98357500$

$-1.41385100$

0.09895700

2.55233700

$-0.47547900$

0.16085700

3.30208700

0.27634900

$-0.10753600$

2.51162300

$-0.55946400$

1.25130600

2.86425200

$-1.43901200$

$-0.25157600$

1.22136300

$-0.02634700$

$-1.48544700$

1.06233200

2.10356800

$-0.41191600$

TS1

C

$\mathrm{H}$

$-2.66487800$

$-1.77047400$

1.12731100

$-2.36154900$

$-1.30663200$

2.07059900

$-1.76745700$

$-2.01440000$

0.55083600

$-3.23228600$

$-2.68089000$

1.34318000

$-3.73154700$

$-0.64530300$

0.14334700

$-2.41030200$

0.19982800

$-0.84776800$

$-2.93831300$

0.82363200

$-1.57833700$

$-1.43489800$

1.04460500

$-0.02203200$

$-1.09194200$

0.44679500

0.82896800

0.06847400

1.38039300

$-1.03410200$ 


$\begin{array}{lrrc}\mathrm{C} & 3.49815800 & -0.09302800 & 0.08625700 \\ \mathrm{C} & 2.37537000 & 0.36913700 & 1.03154100 \\ \mathrm{C} & 0.94663800 & -0.71453200 & -0.50179200 \\ \mathrm{~S} & 2.64756700 & -0.62227800 & -1.44081200 \\ \mathrm{H} & 4.19861300 & 0.72591200 & -0.11002300 \\ \mathrm{O} & 1.27670200 & -0.51277600 & 0.85265400 \\ \mathrm{O} & 0.13626800 & -1.58340100 & -0.78369700 \\ \mathrm{H} & 4.05181900 & -0.93065100 & 0.52907700 \\ \mathrm{H} & 2.05354300 & 1.37620700 & 0.73664700 \\ \mathrm{C} & 2.76820700 & 0.33160100 & 2.49932800 \\ \mathrm{H} & 3.62413000 & 0.99300200 & 2.68169900 \\ \mathrm{H} & 1.93472100 & 0.66287100 & 3.12760200 \\ \mathrm{H} & 3.04286300 & -0.68671000 & 2.79826100 \\ \mathrm{C} & -2.07693000 & 2.32758800 & 0.50347300 \\ \mathrm{H} & -2.35941000 & 2.98518700 & -0.32850900 \\ \mathrm{H} & -2.98154900 & 2.10037600 & 1.08449100 \\ \mathrm{H} & -1.37579000 & 2.87788500 & 1.13978800 \\ \mathrm{H} & -1.86123900 & -0.57650900 & -1.37878700\end{array}$

INT3

$\begin{array}{lrrc}\mathrm{C} & -3.13144600 & -2.21974100 & -0.74365800 \\ \mathrm{H} & -3.75542700 & -2.73743900 & -1.47683700 \\ \mathrm{H} & -2.82350000 & -2.92459100 & 0.03369700 \\ \mathrm{H} & -2.23789100 & -1.82561800 & -1.23130100 \\ \mathrm{~S} & -4.12968400 & -0.86104100 & -0.02728000 \\ \mathrm{C} & -2.76871300 & 0.09993300 & 0.75675300 \\ \mathrm{H} & -2.07174300 & -0.61725100 & 1.19222600 \\ \mathrm{C} & -2.04414700 & 1.02553400 & -0.22673500 \\ \mathrm{H} & -1.77577800 & 0.45947200 & -1.12099500 \\ \mathrm{~S} & -0.44401000 & 1.59608300 & 0.49038100 \\ \mathrm{C} & 0.49120900 & 0.05498300 & 0.26962200 \\ \mathrm{O} & 0.01647800 & -0.94571300 & -0.23424000 \\ \mathrm{O} & 1.69157400 & 0.22727200 & 0.76777600 \\ \mathrm{C} & 2.65927900 & -0.89633400 & 0.65082500 \\ \mathrm{H} & 2.07068900 & -1.80333400 & 0.83977700 \\ \mathrm{C} & 3.66222000 & -0.65325600 & 1.76072300 \\ \mathrm{H} & 3.16522100 & -0.55377800 & 2.73328300 \\ \mathrm{H} & 4.35690900 & -1.50048200 & 1.80244100 \\ \mathrm{H} & 4.23434800 & 0.24667900 & 1.51723200 \\ \mathrm{C} & 3.27020000 & -0.94715900 & -0.75581800 \\ \mathrm{H} & 2.43620700 & -1.10168600 & -1.45512400 \\ \mathrm{H} & -3.21636200 & 0.68186300 & 1.56901200 \\ \mathrm{C} & -2.87731400 & 2.24744100 & -0.60730000 \\ \mathrm{H} & -3.83700100 & 1.91928500 & -1.02103600\end{array}$




$\begin{array}{lrrr}\mathrm{H} & -3.07980800 & 2.87672100 & 0.26816100 \\ \mathrm{H} & -2.36263900 & 2.85761700 & -1.35518400 \\ \mathrm{H} & 3.84158900 & -1.89368600 & -0.76385900 \\ \mathrm{~S} & 4.31263300 & 0.47716800 & -1.23974100\end{array}$

TS2

C

$\mathrm{H}$

$\mathrm{H}$

$\mathrm{H}$

$\mathrm{S}$

C

$\mathrm{H}$

C

$\mathrm{H}$

$\mathrm{S}$

C

$\mathrm{O}$

C

C

$\mathrm{S}$

$\mathrm{H}$

$\mathrm{H}$

$\mathrm{O}$

C

$\mathrm{H}$

$\mathrm{H}$

$\mathrm{H}$

$\mathrm{H}$

C

$\mathrm{H}$

$\mathrm{H}$

$\mathrm{H}$

$\mathrm{H}$

$\mathrm{C}_{3} \mathrm{H}_{6} \mathrm{~S}$

$\mathrm{C}$

C

$\mathrm{H}$

$\mathrm{H}$

$\mathrm{H}$

$\mathrm{S}$

C

$\mathrm{H}$

$\begin{array}{rrr}2.72354300 & 2.52395000 & -0.55963600 \\ 3.19944700 & 3.12614900 & -1.33807300 \\ 2.32301100 & 3.18434200 & 0.21497000 \\ 1.90080300 & 1.94530100 & -0.98549100 \\ 3.98709600 & 1.39436200 & 0.14216800 \\ 2.85816200 & 0.07595100 & 0.76643200 \\ 1.97595700 & 0.57085300 & 1.17368200 \\ 2.45105300 & -0.92972000 & -0.31544200 \\ 2.11766900 & -0.37975000 & -1.19806900 \\ 0.98076700 & -1.89147600 & 0.24055000 \\ -0.33541200 & -0.58157200 & -0.01818100 \\ -0.00937200 & 0.50761100 & -0.48654400 \\ -2.94756200 & 0.22296300 & 0.05921200 \\ -4.09135200 & -0.69150700 & -0.02458500 \\ -5.28933700 & 0.60411300 & -0.57271100 \\ -3.94306600 & -1.47765300 & -0.76973700 \\ -4.34169200 & -1.14052200 & 0.94212800 \\ -1.46574800 & -1.02159200 & 0.35255400 \\ -2.70787400 & 1.08103500 & 1.27264100 \\ -2.79545200 & 0.47500600 & 2.18106700 \\ -1.70667100 & 1.51543200 & 1.22363700 \\ -3.44997200 & 1.88023500 & 1.31346300 \\ -2.55425800 & 0.58740400 & -0.88008100 \\ 3.58505500 & -1.88348000 & -0.68428400 \\ 3.28856200 & -2.55068200 & -1.49946500 \\ 4.46248100 & -1.30864900 & -1.00262800 \\ 3.87506900 & -2.50285600 & 0.17365300 \\ 3.37899200 & -0.43046800 & 1.58581500\end{array}$

0.52070200

0.16641700

0.50475700

$-0.36255000$

1.15615700

$-0.15400900$

0.58745300

0.23945700

1.58916100

$-0.09884200$

1.48245200

$-1.15777800$

$-0.85016600$

1.91349800

0.45481100

$-1.09910200$

$-0.52612600$

$-0.05412500$

1.78057300

$-0.31077700$

$-0.17859200$

2.60397100

0.02143100 
INT4

C

$\mathrm{H}$

$\mathrm{H}$

$\mathrm{H}$

$\mathrm{S}$

C

$\mathrm{H}$

C

H

$\mathrm{S}$

C

O

$\mathrm{O}$

$\mathrm{C}$

$\mathrm{H}$

$\mathrm{H}$

H

H

$\mathrm{CO}_{2}$

$\mathrm{C}$

$\mathrm{O}$

O

TS3

$\mathrm{C}$

$\mathrm{H}$

H

$\mathrm{H}$

$\mathrm{S}$

C

$\mathrm{H}$

C

$\mathrm{H}$

S

C

C

$\mathrm{H}$

H

$\mathrm{H}$

$\begin{array}{ccc}2.13240900 & -1.83344900 & 0.44112800 \\ 2.74845500 & -2.01570300 & 1.32644700 \\ 2.27338500 & -2.65364100 & -0.26986700 \\ 1.07626700 & -1.78852700 & 0.72267000 \\ 2.62566000 & -0.23141700 & -0.31145400 \\ 0.94712100 & 0.35285500 & -0.84004100 \\ 0.42109400 & -0.51194900 & -1.24429800 \\ 0.12486500 & 0.97934300 & 0.28938800 \\ 0.15722400 & 0.30386400 & 1.14795800 \\ -1.64362200 & 1.04927300 & -0.20823600 \\ -2.14173000 & -0.90289000 & 0.06801000 \\ -1.19851400 & -1.58411600 & 0.47775500 \\ -3.31455100 & -1.08261000 & -0.22438200 \\ 0.64817200 & 2.35736900 & 0.68973100 \\ 0.07737700 & 2.75962300 & 1.53295300 \\ 1.70624900 & 2.29624300 & 0.97679100 \\ 0.55917600 & 3.06584200 & -0.14393100 \\ 1.10767200 & 1.07298900 & -1.64996400\end{array}$

$\begin{array}{ccc}0.00000000 & 0.00000000 & -0.00000800 \\ 0.00000000 & 0.00000000 & -1.16916100 \\ 0.00000000 & 0.00000000 & 1.16916700\end{array}$

3.71658600

$-2.11439800$

$-0.38832500$

$4.45745900 \quad-2.68390000$

0.18090000

4.19327800

$-1.72472500$

$-1.29448400$

2.90080300

$-2.78601600$

$-0.67700500$

3.10535700

$-0.75985700$

0.67216000

1.89148400

$-0.00520700$

$-0.52753500$

1.30050500

$-0.82643500$

$-0.94246300$

0.96453900

1.02388500

0.11970400

0.41560300

0.52073500

0.92814300

$-0.23905900$

1.59613900

$-1.15421300$

$-2.96843800$

0.17383100

0.13752400

$-1.96485800$

$-0.42185200$

$-0.73363400$

$-3.60004700$

0.90650900

$-0.36740000$

$-1.11289400$

$-0.93185400$

$-0.31040800$

$-2.08151500$

$-0.42825600$

$-1.80405700$ 
$-3.65702900$

$-1.54081900$

0.00970300

$-2.52287800$

0.64456200

1.50574100

$-1.93151800$

1.56134800

1.39114700

$-3.38642500$

0.83979500

2.15223500

$-1.90402400$

$-0.11939900$

1.98853300

1.74128700

2.19637900

0.72629700

1.04443400

2.91779500

1.16373900

2.44398800

1.86870700

1.50744200

2.31232300

2.71447300

$-0.05359100$

2.45339100

0.46060600

$-1.34376600$

INT5

C

$\mathrm{H}$

$\mathrm{H}$

$\mathrm{H}$

$\mathrm{S}$

C

C

$\mathrm{S}$

C

C

$\mathrm{H}$

$\mathrm{H}$

C

$\mathrm{H}$

$\mathrm{H}$

$\mathrm{H}$

H

$\mathrm{H}$

C

$\mathrm{H}$

$\mathrm{H}$

$\mathrm{H}$

$\mathrm{H}$

$\mathrm{H}$

$\mathrm{S}$

INT6

C

C

C

C

C

$\begin{array}{rrc}4.46338300 & -0.35531200 & -0.09229300 \\ 5.32291100 & -0.90333500 & 0.30466500 \\ 4.39026800 & 0.60712300 & 0.42015500 \\ 4.62002900 & -0.18409900 & -1.16224300 \\ 2.98576900 & -1.39376700 & 0.18863300 \\ 1.68899200 & -0.38761900 & -0.66841400 \\ 0.91140900 & 0.59140000 & 0.21616000 \\ -0.37197800 & 1.43021000 & -0.81267500 \\ -1.73729600 & 0.11807400 & -0.85617000 \\ -2.82613900 & 0.22613100 & 0.21042600 \\ -1.25248800 & -0.86268500 & -0.80821000 \\ -2.17970300 & 0.21604400 & -1.84865700 \\ -2.26361000 & 0.12826900 & 1.63149700 \\ -1.76152500 & -0.83809200 & 1.77068700 \\ -1.55267800 & 0.93982900 & 1.85990300 \\ -3.08415900 & 0.17256800 & 2.35452600 \\ 2.16871800 & 0.14023700 & -1.49951000 \\ 0.38605100 & 0.02833000 & 0.99229100 \\ 1.78823100 & 1.65054100 & 0.88124400 \\ 2.52806300 & 1.17961400 & 1.53866800 \\ 2.31903900 & 2.25094500 & 0.13126100 \\ 1.17334500 & 2.32719200 & 1.48147400 \\ 0.99825900 & -1.11540800 & -1.10055100 \\ -3.29383600 & 1.21689400 & 0.10217600 \\ -4.04704800 & -1.09919700 & -0.15541700\end{array}$

0.34493100

$-0.72346800$

0.32839100

$-0.94272600$

$-1.41766900$

0.72231700

$-2.04636800$

$-1.29012400$

$-0.34767800$

$-2.79569100$

0.04726100

$-0.34105900$

$-0.85384800$

1.44361800

0.59542400 


$\begin{array}{lrrc}\mathrm{C} & -1.91164700 & 1.28845100 & -0.51112500 \\ \mathrm{H} & -1.31339200 & -1.05219900 & 1.68952000 \\ \mathrm{H} & -1.59370600 & -1.46577100 & -1.33247700 \\ \mathrm{H} & -3.33917200 & 0.14097200 & 0.61113400 \\ \mathrm{H} & -1.30358100 & 1.20100400 & 1.56735300 \\ \mathrm{H} & -0.67579100 & -2.46861000 & 0.84650700 \\ \mathrm{H} & -2.77558100 & -2.09530700 & -0.19385900 \\ \mathrm{H} & -3.55870900 & 0.03586100 & -1.12979800 \\ \mathrm{H} & -0.53988900 & 2.48995200 & 0.65784500 \\ \mathrm{H} & -2.55386800 & 2.17912300 & -0.50776100 \\ \mathrm{H} & -1.40382000 & 1.26905200 & -1.48492000 \\ \mathrm{C} & 2.55185700 & -0.86184800 & -0.51071500 \\ \mathrm{C} & 1.51314000 & 1.37018400 & -0.15862300 \\ \mathrm{C} & 2.77719500 & 0.54363600 & 0.04999100 \\ \mathrm{H} & 2.55614100 & -0.82946000 & -1.61148000 \\ \mathrm{H} & 3.38163100 & -1.52050800 & -0.22537700 \\ \mathrm{H} & 1.59731400 & 2.34114500 & 0.34324300 \\ \mathrm{H} & 1.36591400 & 1.57223900 & -1.23294900 \\ \mathrm{H} & 2.98712700 & 0.48148500 & 1.12459600 \\ \mathrm{H} & 3.63279900 & 1.03360200 & -0.42894700 \\ \mathrm{~N} & 0.36468800 & 0.66337700 & 0.40199900 \\ \mathrm{~N} & 1.30895900 & -1.47949600 & -0.06688100\end{array}$

TS4

$\mathrm{C}$

C

$\mathrm{S}$

$\mathrm{H}$

$\mathrm{O}$

C

$\mathrm{O}$

$\mathrm{H}$

C

C

C

C

C

C

$\mathrm{H}$

$\mathrm{H}$

$\mathrm{H}$

$\mathrm{H}$

$\mathrm{H}$

$\mathrm{H}$
$-1.96971800$

$-1.41106400$

$-3.78612900$

$-1.56229100$

$-3.02474600$

$-4.11084300$

$-5.26667200$

$-1.66773200$

1.49342500

1.26708900

1.72539700

3.23482900

3.91549000

4.11948000

1.79525700

1.41081600

3.49132100

3.83320300

0.20861800

1.19635900
0.57348000

0.01348300

0.76099700

1.57404100

$-1.42081100$

$-0.92665100$

$-1.29124100$

$-0.06173100$

0.14383200

$-1.26606200$

$-2.33408800$

$-2.60029000$

$-0.25592500$

$-1.36571000$

$-1.40527500$

$-2.02733100$

$-3.04265800$

$-0.69020600$

$-1.38464300$

$-3.26808700$
$-0.86378000$

0.42386200

$-0.81981000$

$-1.03292000$

0.47562300

0.05097600

0.10181300

$-1.70137400$

$-0.31658600$

$-0.81609400$

0.20000100

0.16955700

$-0.65903400$

0.38459700

$-1.76596700$

1.20585400

$-0.80394900$

$-1.66301700$

$-1.03959100$

$-0.01797800$ 


$\begin{array}{lrrr}\mathrm{H} & 3.48230100 & -3.35556900 & 0.92472300 \\ \mathrm{H} & 4.79255200 & 0.39521800 & -0.67724000 \\ \mathrm{H} & 5.16999800 & -1.67942800 & 0.34560500 \\ \mathrm{H} & 3.95553500 & -0.94148500 & 1.38431600 \\ \mathrm{C} & 0.66403400 & 2.18351500 & 0.63275500 \\ \mathrm{C} & 3.07704600 & 1.97017700 & 0.09797000 \\ \mathrm{C} & 1.85228800 & 2.86277100 & -0.04025200 \\ \mathrm{H} & 0.78796800 & 2.18816700 & 1.72377900 \\ \mathrm{H} & -0.25860700 & 2.73383400 & 0.41998100 \\ \mathrm{H} & 3.91059500 & 2.36333700 & -0.49267000 \\ \mathrm{H} & 3.40785400 & 1.92610500 & 1.14649900 \\ \mathrm{H} & 1.63408300 & 3.01970400 & -1.10277600 \\ \mathrm{H} & 2.04579100 & 3.84134800 & 0.40995900 \\ \mathrm{~N} & 2.77029500 & 0.62465800 & -0.39385800 \\ \mathrm{~N} & 0.48816000 & 0.81223700 & 0.17005100 \\ \mathrm{C} & -1.71633100 & 0.64556900 & 1.75187900 \\ \mathrm{H} & -0.90151700 & 0.47002200 & 2.45920900 \\ \mathrm{H} & -2.62010500 & 0.18098800 & 2.14714200 \\ \mathrm{H} & -1.90361800 & 1.71792900 & 1.65824600 \\ \mathrm{H} & -0.95488300 & -0.95667000 & 0.44118800\end{array}$

\section{INT7}

C

$\mathrm{C}$

C

C

C

$\mathrm{H}$

H

$\mathrm{H}$

$\mathrm{H}$

$\mathrm{H}$

$\mathrm{H}$

$\mathrm{H}$

$\mathrm{H}$

$\mathrm{H}$

$\mathrm{H}$

$\mathrm{N}$

C

C

$\mathrm{N}$

C

C

$\mathrm{H}$
$-0.79815300$

$-2.04618400$

$-2.03370200$

$-3.30139900$

$-3.09651400$

$-0.08883500$

$-2.47968100$

$-1.63216200$

$-3.58210000$

$-1.05865300$

$-2.34989900$

$-1.71110800$

$-4.14836100$

$-4.04896200$

$-2.80643100$

$-1.01921400$

$-0.40138000$

$-0.09187300$

1.13699800

1.71011000

0.66230600

0.05868600
$-1.18237400$

1.25185000

$-1.88204900$

0.33214700

$-0.95072900$

$-1.95951000$

$-2.46435400$

1.32638000

0.08342800

$-0.66120800$

2.26640800

$-2.60185600$

0.88423900

$-1.49055500$

$-0.67302700$

0.85576600

1.97660100

$-0.16529500$

0.32879400

1.56155500

2.62249000

1.59623700
$-0.98214600$

$-0.62243000$

$-0.39829100$

$-0.60798600$

0.20165200

$-1.26601400$

$-1.21584600$

$-1.63718300$

$-1.63993900$

$-1.91047400$

$-0.35261500$

0.36146600

$-0.18337000$

0.27586300

1.21892800

0.35550600

1.05781500

$-0.06422800$

$-0.70802200$

$-0.12982300$

0.17794200

1.97664900 


$\begin{array}{lrrr}\mathrm{H} & -1.18783500 & 2.67955600 & 1.34690600 \\ \mathrm{H} & 0.20836500 & 2.99734700 & -0.74771400 \\ \mathrm{H} & 1.14710400 & 3.46982900 & 0.67807800 \\ \mathrm{H} & 2.25124600 & 1.34531800 & 0.80705800 \\ \mathrm{H} & 2.44219200 & 1.94773400 & -0.84472700 \\ \mathrm{C} & 2.12799500 & -0.76007800 & -0.68237200 \\ \mathrm{C} & 2.21134900 & -1.32110300 & 0.77010000 \\ \mathrm{H} & 2.50920700 & -2.37299800 & 0.75541000 \\ \mathrm{H} & 2.94694400 & -0.76757200 & 1.36285700 \\ \mathrm{~S} & 0.54809800 & -1.12802700 & 1.50566400 \\ \mathrm{C} & 3.49812900 & -0.38126200 & -1.24113200 \\ \mathrm{H} & 4.03397100 & 0.32482800 & -0.60007700 \\ \mathrm{H} & 4.11433000 & -1.28277300 & -1.32493200 \\ \mathrm{H} & 3.40056300 & 0.05771100 & -2.23952700 \\ \mathrm{H} & 1.74325600 & -1.55916400 & -1.31842300\end{array}$

TS5

$\mathrm{C}$

C

C

$\mathrm{O}$

C

C

C

C

C

C

$\mathrm{H}$

$\mathrm{H}$

H

$\mathrm{H}$

$\mathrm{H}$

$\mathrm{H}$

H

H

$\mathrm{H}$

$\mathrm{H}$

C

C

C

$\mathrm{H}$

H

H

$\mathrm{H}$

$\begin{array}{rrc}-2.11732400 & -1.10003100 & 0.60044200 \\ -1.34288700 & -0.77898000 & -0.67536000 \\ -3.59966400 & 0.73920300 & 0.18400700 \\ -4.24359700 & 1.66190300 & 0.61943600 \\ 1.31652900 & -0.18916000 & 0.21589800 \\ 0.70805600 & 1.09155900 & 0.74394300 \\ 0.86886500 & 2.27460200 & -0.23705300 \\ 2.24277000 & 2.95001700 & -0.17808900 \\ 3.54306400 & 0.84919000 & 0.54575800 \\ 3.43461600 & 2.02149000 & -0.44111200 \\ 1.16901800 & 1.34285600 & 1.70633900 \\ 0.65980500 & 1.92840100 & -1.25768300 \\ 2.36846700 & 3.40160300 & 0.81664400 \\ 3.34694900 & 1.19523400 & 1.56787600 \\ -0.34816600 & 0.92185000 & 0.94776200 \\ 0.09752700 & 3.01505500 & -0.00113700 \\ 2.26767400 & 3.77808200 & -0.89623200 \\ 4.56347100 & 0.45886500 & 0.54265600 \\ 4.35874700 & 2.60890100 & -0.37330000 \\ 3.39250500 & 1.61829400 & -1.46186900 \\ 1.04925200 & -2.36066400 & -0.78698600 \\ 3.32639800 & -1.48314100 & -0.32868400 \\ 2.41370400 & -2.69574000 & -0.19413500 \\ 1.11511600 & -2.27925900 & -1.88153600 \\ 0.32641600 & -3.15544800 & -0.57222600 \\ 4.26076400 & -1.64497800 & 0.21770700 \\ 3.58776000 & -1.31101300 & -1.38326200\end{array}$




$\begin{array}{lrrc}\mathrm{H} & 2.29995100 & -2.94953900 & 0.86595000 \\ \mathrm{H} & 2.85898900 & -3.55835100 & -0.69965100 \\ \mathrm{~N} & 2.67610000 & -0.29367900 & 0.22959000 \\ \mathrm{~N} & 0.52476700 & -1.11841500 & -0.23993000 \\ \mathrm{H} & -1.11714800 & 0.23720000 & -0.93649700 \\ \mathrm{H} & -1.33011800 & -1.49890700 & -1.48175400 \\ \mathrm{O} & -2.72759500 & 0.09407100 & 1.08339500 \\ \mathrm{~S} & -3.56391800 & 0.08512100 & -1.45111500 \\ \mathrm{C} & -1.35289100 & -1.69595500 & 1.78148100 \\ \mathrm{H} & -2.07193300 & -1.90237600 & 2.57956300 \\ \mathrm{H} & -0.86972000 & -2.63600900 & 1.49781100 \\ \mathrm{H} & -0.59310200 & -1.01692700 & 2.17210600 \\ \mathrm{H} & -2.89976400 & -1.81294600 & 0.31763100\end{array}$

INT8

$\begin{array}{lrrr}\mathrm{C} & -0.63284800 & 0.64433400 & 0.07217700 \\ \mathrm{C} & -1.32070900 & 0.75245400 & -1.26644600 \\ \mathrm{C} & -2.78453800 & 1.23480200 & -1.14334500 \\ \mathrm{C} & -3.78430800 & 0.11290200 & -0.85362900 \\ \mathrm{C} & -2.13373000 & -1.22244700 & 0.58860500 \\ \mathrm{C} & -3.53391700 & -0.61737400 & 0.46691800 \\ \mathrm{H} & -1.24222900 & -0.21564700 & -1.77065000 \\ \mathrm{H} & -2.85937700 & 2.02064100 & -0.37895200 \\ \mathrm{H} & -3.73590200 & -0.61574500 & -1.67396500 \\ \mathrm{H} & -1.80605100 & -1.73701500 & -0.32035400 \\ \mathrm{H} & -0.76807700 & 1.44632200 & -1.89050000 \\ \mathrm{H} & -3.05283400 & 1.70673300 & -2.09526600 \\ \mathrm{H} & -4.80238200 & 0.52112800 & -0.85600700 \\ \mathrm{H} & -2.12595700 & -1.96263600 & 1.38936400 \\ \mathrm{H} & -4.25841700 & -1.43478600 & 0.56596100 \\ \mathrm{H} & -3.70459600 & 0.05739200 & 1.31724300 \\ \mathrm{C} & 0.70251700 & 1.65408400 & 1.83297900 \\ \mathrm{C} & -0.46080800 & -0.49998000 & 2.26929900 \\ \mathrm{C} & 0.81776900 & 0.29085000 & 2.49131500 \\ \mathrm{H} & -0.06615000 & 2.26706700 & 2.32266000 \\ \mathrm{H} & 1.64605000 & 2.19677700 & 1.88510400 \\ \mathrm{H} & -0.22523700 & -1.56695400 & 2.29268000 \\ \mathrm{H} & -1.21969300 & -0.28223500 & 3.03134700 \\ \mathrm{H} & 1.64114600 & -0.26431100 & 2.03467900 \\ \mathrm{H} & 1.00582900 & 0.39730800 & 3.56433400 \\ \mathrm{~N} & -1.08368200 & -0.24178400 & 0.95322800 \\ \mathrm{~N} & 0.33499700 & 1.51845500 & 0.41410400 \\ \mathrm{C} & 1.25199000 & 2.05819700 & -0.59885300 \\ \mathrm{C} & 2.21578800 & 0.98294800 & -1.18290200\end{array}$




$\begin{array}{lrrc}\mathrm{O} & 0.13029300 & -1.87299300 & -1.62116700 \\ \mathrm{C} & 1.11091900 & -1.38575800 & -1.07015600 \\ \mathrm{H} & 0.67619300 & 2.50528800 & -1.40932700 \\ \mathrm{O} & 1.42168000 & -0.10206000 & -1.64560900 \\ \mathrm{~S} & 2.04538600 & -2.05878400 & 0.20146400 \\ \mathrm{H} & 1.81808400 & 2.86616800 & -0.12985100 \\ \mathrm{C} & 3.43793100 & 0.73340800 & -0.29830200 \\ \mathrm{H} & 3.18910500 & 0.45390400 & 0.72336700 \\ \mathrm{H} & 4.04658600 & -0.07349400 & -0.70763200 \\ \mathrm{H} & 4.02953300 & 1.65967600 & -0.28830200 \\ \mathrm{H} & 2.59687500 & 1.42816700 & -2.11243800\end{array}$

COS

$\mathrm{C}$

$\mathrm{O}$

$\mathrm{S}$

$\begin{array}{rrr}0.00000000 & 0.00000000 & -0.52902000 \\ 0.00000000 & 0.00000000 & -1.69389100 \\ 0.00000000 & 0.00000000 & 1.04532800\end{array}$

INT9

$\mathrm{C}$

C

C

C

C

C

$\mathrm{H}$

$\mathrm{H}$

$\mathrm{H}$

$\mathrm{H}$

$\mathrm{H}$

$\mathrm{H}$

$\mathrm{H}$

$\mathrm{H}$

$\mathrm{H}$

$\mathrm{H}$

C

C

C

$\mathrm{H}$

$\mathrm{H}$

$\mathrm{H}$

$\mathrm{H}$

$\mathrm{H}$

$\mathrm{H}$

$\mathrm{N}$

$-0.03914000$

$-0.30179300$

0.82049800

$-1.56763300$

0.10899600

2.08289100

$-1.40367100$

0.96645500

3.29905900

$-0.92771100$

0.15976900

1.87860800

0.72490300

$-1.22565600$

3.17167000

0.47717100

$-0.43861000$

1.08119300

$-1.93206000$

$-0.89160400$

1.86761700

$-0.71661300$

1.79369900

3.47047600

$-1.64668400$

$-0.65504300$

1.72520300

$-0.05136200$

$-1.98946500$

0.16934800

$-2.32642000$

0.55430500

2.33107500

$-2.37081000$

1.42126500

4.19558000

$-0.96390300$

0.79240100

1.98120000

1.66644100

$-1.77436200$

4.03100600

0.66114600

$-1.09837800$

3.21437200

1.22781300

0.36118000

$-0.88430900$

1.31082500

1.62871400

$-0.09128000$

2.06440800

$-0.61184700$

$-1.20539500$

2.22083600

0.44097800

0.07537400

1.60021300

2.06854700

$-1.63974000$

1.39278000

2.41607100

$-0.51753900$

2.05100400

$-1.62643200$

0.59406900

2.91768300

$-0.55499300$

$-2.17298800$

1.93032100

0.01727000

$-1.29338900$

3.26730100

0.75591900

0.71501300

0.86554300

$-0.34683500$ 


$\begin{array}{lrrr}\mathrm{N} & -0.80494900 & -0.09527800 & 1.22246900 \\ \mathrm{C} & -2.10594200 & -0.74640500 & 1.03379100 \\ \mathrm{C} & -2.13763300 & -1.21783200 & -0.44623300 \\ \mathrm{H} & -2.23646500 & -1.59267200 & 1.71943600 \\ \mathrm{O} & -1.04441600 & -0.55079200 & -1.05708900 \\ \mathrm{H} & -2.92222400 & -0.03959700 & 1.22850300 \\ \mathrm{H} & -1.97957000 & -2.30696500 & -0.49143600 \\ \mathrm{C} & -3.41743100 & -0.86891100 & -1.18791100 \\ \mathrm{H} & -4.27709400 & -1.37821200 & -0.73699500 \\ \mathrm{H} & -3.59256700 & 0.21208300 & -1.15305300 \\ \mathrm{H} & -3.34963600 & -1.17212900 & -2.23748200\end{array}$

TS6

C

C

S

H

O

C

$\mathrm{H}$

C

$\mathrm{H}$

$\mathrm{H}$

$\mathrm{H}$

O

$\mathrm{H}$

C

C

C

C

C

C

$\mathrm{H}$

H

$\mathrm{H}$

H

$\mathrm{H}$

H

$\mathrm{H}$

$\mathrm{H}$

H

$\mathrm{H}$

C

\begin{tabular}{ccc}
-3.32446500 & -0.05991800 & -1.12300800 \\
-3.07688600 & -1.15435900 & -0.08029000 \\
-2.53619500 & 1.47243600 & -0.52711300 \\
-4.40273400 & 0.08455500 & -1.25246600 \\
-1.68455600 & -1.07574200 & 0.28354300 \\
-3.33117800 & -2.56445600 & -0.58221800 \\
-3.66923100 & -0.94726900 & 0.81827300 \\
-1.39456600 & 0.09644000 & 0.96604500 \\
-4.37654600 & -2.66535300 & -0.89312900 \\
-2.69328400 & -2.78662600 & -1.44524100 \\
-3.12995400 & -3.30239100 & 0.20083700 \\
-1.71465600 & 0.29480700 & 2.12535300 \\
-2.90287500 & -0.37447600 & -2.08618700 \\
0.98337900 & 0.02221900 & -0.10754400 \\
1.01555800 & -1.46392500 & -0.35223600 \\
1.97306000 & -2.18444500 & 0.62555700 \\
3.44704800 & -2.13404500 & 0.21310000 \\
3.27096500 & 0.10607300 & -1.03832200 \\
4.01093600 & -0.72230600 & 0.02078000 \\
1.31339400 & -1.65774900 & -1.38859400 \\
1.83756600 & -1.76319700 & 1.62981200 \\
3.56610900 & -2.68504700 & -0.73112100 \\
3.05550900 & -0.50321600 & -1.92418800 \\
0.00805500 & -1.84245800 & -0.22500800 \\
1.66019500 & -3.23256700 & 0.68819600 \\
4.05171200 & -2.66569600 & 0.95769600 \\
3.91227000 & 0.92339200 & -1.37475800 \\
5.06169600 & -0.80015700 & -0.28459900 \\
3.99761500 & -0.16803100 & 0.96849000 \\
0.20475100 & 1.98187600 & 1.05018500 \\
& & \\
\hline
\end{tabular}




$\begin{array}{lrrr}\mathrm{C} & 2.09145800 & 2.21761400 & -0.50502300 \\ \mathrm{C} & 0.75280400 & 2.80707700 & -0.10140200 \\ \mathrm{H} & 0.89400100 & 1.97644700 & 1.90397800 \\ \mathrm{H} & -0.76231700 & 2.33352500 & 1.40033100 \\ \mathrm{H} & 2.39588800 & 2.56831600 & -1.49793200 \\ \mathrm{H} & 2.87692200 & 2.51220000 & 0.20443200 \\ \mathrm{H} & 0.03359200 & 2.76147900 & -0.92519400 \\ \mathrm{H} & 0.88220400 & 3.85382100 & 0.18967800 \\ \mathrm{~N} & 2.03938600 & 0.74677800 & -0.55045400 \\ \mathrm{~N} & 0.02145000 & 0.59349600 & 0.59553800\end{array}$

INT10

$\begin{array}{lrrc}\mathrm{C} & 1.58526600 & -0.87148300 & 1.03249800 \\ \mathrm{C} & 2.10094600 & 1.22601700 & -1.34646100 \\ \mathrm{O} & 2.25792800 & -1.82322600 & 1.37356800 \\ \mathrm{C} & 2.97161900 & 0.56991600 & -0.27877200 \\ \mathrm{O} & 2.12155300 & 0.38199500 & 0.87317300 \\ \mathrm{C} & -0.42236500 & 0.06624100 & -0.10295600 \\ \mathrm{C} & -0.58353600 & 1.44954900 & 0.55047300 \\ \mathrm{C} & -1.69552500 & 1.51293200 & 1.61488800 \\ \mathrm{C} & -3.06377700 & 1.90366700 & 1.03941000 \\ \mathrm{C} & -2.69868700 & 0.41246700 & -1.02614400 \\ \mathrm{C} & -3.66717100 & 0.88937400 & 0.06232500 \\ \mathrm{H} & -0.77184600 & 2.19627800 & -0.22749400 \\ \mathrm{H} & -1.75507400 & 0.55561100 & 2.14633100 \\ \mathrm{H} & -2.95246000 & 2.86938700 & 0.52487800 \\ \mathrm{H} & -2.23749300 & 1.25621100 & -1.55539800 \\ \mathrm{H} & 0.37505800 & 1.69677100 & 0.99975300 \\ \mathrm{H} & -1.41062100 & 2.26311400 & 2.36243000 \\ \mathrm{H} & -3.77239400 & 2.07427500 & 1.85986900 \\ \mathrm{H} & -3.25769200 & -0.14254400 & -1.78577500 \\ \mathrm{H} & -4.55253800 & 1.33169200 & -0.41440700 \\ \mathrm{H} & -4.00960400 & 0.00125100 & 0.60925500 \\ \mathrm{C} & -0.43674700 & -2.18736400 & 1.01419400 \\ \mathrm{C} & -1.69406400 & -1.83143200 & -1.09203300 \\ \mathrm{C} & -0.73615500 & -2.78033500 & -0.35674100 \\ \mathrm{H} & -1.35696400 & -2.03917600 & 1.58738300 \\ \mathrm{H} & 0.24569700 & -2.81275400 & 1.58617600 \\ \mathrm{H} & -1.46465000 & -1.79588900 & -2.16830600 \\ \mathrm{H} & -2.72344700 & -2.19398900 & -0.99388000 \\ \mathrm{H} & 0.20754500 & -2.88174700 & -0.90140500 \\ \mathrm{H} & -1.17799000 & -3.77843500 & -0.26669300 \\ \mathrm{~N} & -1.67900400 & -0.49284000 & -0.48580600 \\ & 0.22463900 & -0.88939600 & 0.83081000\end{array}$




$\begin{array}{lrrr}\mathrm{C} & 4.14941700 & 1.43072300 & 0.14754400 \\ \mathrm{H} & 3.79841400 & 2.38673600 & 0.55085800 \\ \mathrm{H} & 4.72677600 & 0.91883200 & 0.92235300 \\ \mathrm{H} & 4.80551900 & 1.63302200 & -0.70600600 \\ \mathrm{H} & 3.31808500 & -0.41145400 & -0.61939700 \\ \mathrm{H} & 1.80468900 & 2.22652000 & -1.01480900 \\ \mathrm{~S} & 0.61239100 & 0.23598300 & -1.75918200 \\ \mathrm{H} & 2.65212600 & 1.33443000 & -2.28711100\end{array}$

INT11

$\begin{array}{lrrc}\mathrm{C} & 1.45544700 & -1.65795600 & 0.18430800 \\ \mathrm{C} & 1.54949700 & 1.84498900 & 0.30374000 \\ \mathrm{O} & 1.62624100 & -2.86370600 & 0.15740600 \\ \mathrm{C} & 2.73768400 & 0.90586300 & 0.10900000 \\ \mathrm{C} & -0.29809100 & 0.20405900 & -0.03922800 \\ \mathrm{C} & -0.56681900 & 0.15535400 & 1.47699700 \\ \mathrm{C} & -1.67187000 & -0.82673900 & 1.90159300 \\ \mathrm{C} & -3.06186900 & -0.17664800 & 1.94759900 \\ \mathrm{C} & -2.60276400 & 1.07960500 & -0.25535200 \\ \mathrm{C} & -3.60327800 & 0.28221000 & 0.58955800 \\ \mathrm{H} & -0.82338100 & 1.16715900 & 1.80876000 \\ \mathrm{H} & -1.67277200 & -1.69848900 & 1.23732400 \\ \mathrm{H} & -3.00891600 & 0.68979200 & 2.62317000 \\ \mathrm{H} & -2.19635500 & 1.93701900 & 0.29722900 \\ \mathrm{H} & 0.36515100 & -0.09800500 & 1.97987200 \\ \mathrm{H} & -1.43364200 & -1.20189900 & 2.90375900 \\ \mathrm{H} & -3.77773300 & -0.87384400 & 2.40101400 \\ \mathrm{H} & -3.12857200 & 1.50633200 & -1.11487400 \\ \mathrm{H} & -4.50567800 & 0.88842700 & 0.74796300 \\ \mathrm{H} & -3.90370600 & -0.59069200 & -0.00386800 \\ \mathrm{C} & -0.15624100 & -1.77933400 & -1.59727600 \\ \mathrm{C} & -1.39398400 & 0.28839900 & -2.23730600 \\ \mathrm{C} & -0.41658200 & -0.79381800 & -2.72867900 \\ \mathrm{H} & -1.07831200 & -2.27968300 & -1.28288200 \\ \mathrm{H} & 0.56262600 & -2.54556600 & -1.88486800 \\ \mathrm{H} & -1.07891100 & 1.28697700 & -2.57398300 \\ \mathrm{H} & -2.39186200 & 0.10205700 & -2.64760800 \\ \mathrm{H} & 0.53831200 & -0.34605600 & -3.02266700 \\ \mathrm{H} & -0.82097700 & -1.31534200 & -3.60281100 \\ \mathrm{H} & -1.53420400 & 0.21849500 & -0.77809700 \\ \mathrm{~S} & 0.43464200 & -1.02000900 & -0.48317800 \\ & 2.85358200 & 0.67997400 & -0.95522500 \\ \mathrm{H} & 1.32489300 & 1.92313600 & 1.37568800 \\ \mathrm{H} & & -0.66433600 & 1.05330200\end{array}$




$\begin{array}{lrrr}\mathrm{O} & 0.40177200 & 1.40276600 & -0.41278000 \\ \mathrm{H} & 3.65526400 & 1.39366000 & 0.45446200 \\ \mathrm{C} & 1.85620200 & 3.23407300 & -0.24774800 \\ \mathrm{H} & 0.97772500 & 3.87731200 & -0.14277400 \\ \mathrm{H} & 2.69383100 & 3.69093100 & 0.28954500 \\ \mathrm{H} & 2.11081800 & 3.17394600 & -1.31136600\end{array}$

TS7

$\mathrm{C}$

$\mathrm{H}$

$\mathrm{H}$

H

$\mathrm{S}$

C

$\mathrm{H}$

C

$\mathrm{H}$

S

C

C

$\mathrm{H}$

$\mathrm{H}$

$\mathrm{S}$

$\mathrm{H}$

C

$\mathrm{H}$

H

$\mathrm{H}$

C

$\mathrm{H}$

$\mathrm{H}$

$\mathrm{H}$

$\mathrm{H}$

\section{INT12}

C

$\mathrm{H}$

$\mathrm{H}$

$\mathrm{H}$

S

C

$\mathrm{H}$

C

S

$\begin{array}{rrc}4.56162900 & -0.49654500 & -1.27541200 \\ 5.36580500 & -1.23846200 & -1.26748100 \\ 4.99491200 & 0.49347600 & -1.09500300 \\ 4.08741200 & -0.50069300 & -2.26305700 \\ 3.36449300 & -0.94046000 & 0.02946200 \\ 2.15566300 & 0.45446800 & -0.24178500 \\ 1.91687700 & 0.47207900 & -1.30831000 \\ 0.86725300 & 0.31141100 & 0.56892900 \\ 0.39106400 & -0.63607100 & 0.27662900 \\ -0.25083900 & 1.69877800 & 0.10091600 \\ -2.62312100 & -0.41312700 & 0.77249400 \\ -2.33949600 & 0.12295400 & -0.55008800 \\ -3.13936000 & 0.27670400 & 1.44183900 \\ -1.58631000 & -0.36833100 & -1.14717000 \\ -3.73425300 & -1.57086700 & -0.14297300 \\ -1.78709100 & -0.90289400 & 1.27064800 \\ -3.16257800 & 1.20940800 & -1.19192000 \\ -2.51803700 & 2.05391400 & -1.45062700 \\ -3.64930800 & 0.84867800 & -2.10370100 \\ -3.94316600 & 1.55279100 & -0.50596600 \\ 1.13330500 & 0.26948300 & 2.07719000 \\ 0.18683900 & 0.18288100 & 2.61927100 \\ 1.77972800 & -0.57438600 & 2.36220600 \\ 1.61781700 & 1.19900300 & 2.39945700 \\ 2.65647500 & 1.39376700 & 0.01633600\end{array}$

$\begin{array}{ccc}-2.81928000 & 1.34437800 & 0.45000800 \\ -2.98705400 & 1.01884900 & 1.48165300 \\ -3.57251500 & 2.09078200 & 0.18147400 \\ -1.81267200 & 1.77808900 & 0.36851800 \\ -2.96999700 & -0.06620200 & -0.71457600 \\ -1.19627200 & -0.55522400 & -0.88189300 \\ -0.65961100 & 0.29523600 & -1.30989600 \\ -0.50643600 & -0.95410800 & 0.42297100 \\ 1.21563600 & -1.53813500 & 0.07314600\end{array}$


C

C

$\mathrm{H}$

$\mathrm{H}$

$\mathrm{C}$

$\mathrm{H}$

$\mathrm{H}$

$\mathrm{H}$

$\mathrm{H}$

$\mathrm{H}$

$\mathrm{S}$

C

$\mathrm{H}$

$\mathrm{H}$

$\mathrm{H}$

$\mathrm{H}$

TS8

$\mathrm{C}$

$\mathrm{H}$

$\mathrm{H}$

$\mathrm{H}$

$\mathrm{S}$

C

C

S

$\mathrm{C}$

C

C

$\mathrm{O}$

$\mathrm{H}$

$\mathrm{H}$

$\mathrm{O}$

$\mathrm{S}$

$\mathrm{H}$

$\mathrm{H}$

C

$\mathrm{H}$

$\mathrm{H}$

$\mathrm{H}$

$\mathrm{H}$

$\mathrm{H}$

C

$\mathrm{H}$
2.16119600

2.06760400

2.00514500

$-1.19901900$

$-1.20638800$

$-2.24140000$

$-1.23125700$

$-0.68917000$

$-0.41188800$

3.03452200

0.71928700

3.60509000

3.67242700

4.21466000

4.03886400

1.76707700

$-4.08921600$

$-5.01994300$

$-3.24414300$

$-4.06705200$

$-4.04652600$

$-2.41859200$

$-2.05361000$

$-0.61197400$

2.33580900

0.98594700

3.43073400

4.39392700

0.69302500

0.33680100

3.34855700

2.06903300

$-1.65779800$

$-2.48665000$

$-1.82666800$

$-2.72010600$

$-1.00251700$

$-1.56291400$

$-2.89337800$

2.59422400

2.46939200

3.51342900
$-0.02172500$

$-0.43952200$

$1.16066500 \quad 0.55056800$

$0.74738700 \quad 1.56807900$

$-1.39101700$

$-1.59000100$

$-2.08004900$

1.18211700

$-1.79701500$

1.39834000

$-3.00249600$

0.58682100

$-2.29518900$

2.12284200

$-0.05920500$

1.04457700

1.68665600

0.49969800

2.36547800

0.20921200

$-0.50922600$

$-0.62278900$

$-1.36050900$

$-1.31156700$

0.30832300

$-1.02645000$

$-0.81302300$

0.33782100

0.30659600

$-1.40518300$

$$
\begin{array}{cc}
2.16063600 & 0.83908300 \\
2.69813100 & 0.63591400 \\
2.82379300 & 0.62758600 \\
1.87890800 & 1.89728600 \\
0.68558700 & -0.23585700 \\
-0.00503600 & 0.32113800 \\
-1.29925400 & -0.41361000 \\
-2.12042000 & 0.40266000 \\
-0.74925900 & 0.71712200 \\
-0.14866200 & 0.36673900 \\
1.08672000 & -0.39933000 \\
1.51329900 & -1.00049000 \\
-0.05128500 & -0.66296600 \\
0.21365900 & 1.13859500 \\
-0.27575000 & -0.19341500 \\
2.00263200 & 0.24841300 \\
0.76344700 & 0.14990600 \\
-0.19759500 & 1.39519800 \\
-1.07171200 & -1.91218800 \\
-0.65857800 & -2.40017700 \\
-0.36851700 & -2.07880600 \\
-2.01555100 & -2.39752400 \\
-1.99671300 & -0.29395600 \\
-0.39524800 & 1.72399500 \\
-2.26764500 & 0.67582500 \\
-2.53219700 & 0.88001800
\end{array}
$$




\section{INT13}

$\mathrm{C}$

$\mathrm{H}$

$\mathrm{H}$

$\mathrm{H}$

S

C

$\mathrm{H}$

$\mathrm{C}$

$\mathrm{S}$

C

C

$\mathrm{H}$

$\mathrm{C}$

$\mathrm{O}$

O

S

$\mathrm{H}$

$\mathrm{H}$

$\mathrm{C}$

$\mathrm{H}$

$\mathrm{H}$

$\mathrm{H}$

$\mathrm{H}$

$\mathrm{H}$

$\mathrm{C}$

$\mathrm{H}$

H

$\mathrm{H}$

INT14

C

$\mathrm{H}$

H

H

S

C

$\mathrm{H}$

C

$\mathrm{S}$

$\begin{array}{ccc}6.02373300 & 0.07058600 & -0.71616200 \\ 6.90600500 & -0.57183500 & -0.78180500 \\ 6.23190100 & 0.88437800 & -0.01392500 \\ 5.81695100 & 0.49053300 & -1.70591700 \\ 4.63546700 & -0.96202700 & -0.13689400 \\ 3.32853200 & 0.33626000 & -0.13210400 \\ 3.34222900 & 0.83349300 & -1.10691400 \\ 1.94172600 & -0.25554800 & 0.12764300 \\ 0.75081000 & 1.14986100 & -0.02671800 \\ -0.83175400 & 0.20748700 & -0.12581700 \\ -2.01498500 & 1.14685300 & -0.41512900 \\ -1.01884600 & -0.32905500 & 0.80234000 \\ -3.75373800 & -0.51364400 & 0.07062100 \\ -3.22412200 & -0.67468200 & 1.17031000 \\ -3.15767600 & 0.40246500 & -0.83908400 \\ -5.15882100 & -1.25124900 & -0.59568500 \\ -0.75141300 & -0.50880200 & -0.94961700 \\ 3.57149200 & 1.07780700 & 0.63818700 \\ -2.32615800 & 2.11397100 & 0.72913200 \\ -2.56793300 & 1.55444300 & 1.63352900 \\ -1.47288900 & 2.77554800 & 0.92374500 \\ -3.18983700 & 2.72890200 & 0.45433300 \\ -1.75987700 & 1.73504800 & -1.30658700 \\ 1.70567300 & -0.96285900 & -0.67603800 \\ 1.83129000 & -0.95789900 & 1.48092400 \\ 0.81175400 & -1.31242900 & 1.65175800 \\ 2.50438900 & -1.82283200 & 1.53057500 \\ 2.09362700 & -0.26834100 & 2.29063700\end{array}$

$\begin{array}{rrr}-4.62921200 & -1.01962900 & -0.28687300 \\ -5.63129700 & -0.66627100 & -0.54618200 \\ -4.66157100 & -1.46797700 & 0.71178900 \\ -4.32407900 & -1.78145200 & -1.01203700 \\ -3.50350700 & 0.41643100 & -0.32916500 \\ -1.93718800 & -0.45952100 & 0.12486400 \\ -1.83050800 & -1.31774900 & -0.54551000 \\ -0.70994600 & 0.44248600 & 0.00640300 \\ 0.76360300 & -0.62569900 & 0.33915400\end{array}$


$\mathrm{O}$

$\mathrm{H}$

$\mathrm{H}$

C

$\mathrm{H}$

$\mathrm{H}$

H

$\mathrm{H}$

$\mathrm{H}$

C

$\mathrm{H}$

$\mathrm{H}$

$\mathrm{H}$

TS9

C

$\mathrm{H}$

$\mathrm{H}$

$\mathrm{H}$

$\mathrm{S}$

C

$\mathrm{H}$

C

S

C

C

C

S

$\mathrm{H}$

$\mathrm{O}$

O

$\mathrm{H}$

$\mathrm{H}$

C

$\mathrm{H}$

$\mathrm{H}$

H

C

$\mathrm{H}$

$\mathrm{H}$

$\mathrm{H}$

$\begin{array}{rcc}2.18004400 & 0.50375100 & -0.16781400 \\ 3.54109500 & -0.18855100 & -0.57108200 \\ 2.38531600 & 1.19876700 & 0.65369800 \\ 4.38331800 & 0.76386500 & -0.91911800 \\ 1.83346100 & 1.07258200 & -1.03695000 \\ -2.03395700 & -0.83083000 & 1.15161300 \\ 4.02921100 & -1.07446900 & 0.61756600 \\ 4.20344000 & -0.43680800 & 1.49650800 \\ 3.33152300 & -1.88196500 & 0.89834600 \\ 4.99157800 & -1.52415500 & 0.33992200 \\ 3.24236300 & -0.93245000 & -1.38332400 \\ -0.62809900 & 0.78829300 & -1.03159300 \\ -0.76890000 & 1.65351700 & 0.93813300 \\ 0.15342400 & 2.23587500 & 0.85863600 \\ -1.61470200 & 2.30977600 & 0.69217800 \\ -0.87559700 & 1.32622400 & 1.97884000\end{array}$

$\begin{array}{rcc}6.01401400 & 0.27292600 & 0.59689200 \\ 6.93950600 & 0.50330700 & 0.06112300 \\ 6.12672100 & -0.69704400 & 1.09301900 \\ 5.84357500 & 1.04369000 & 1.35624000 \\ 4.65278200 & 0.23733900 & -0.61883500 \\ 3.26070300 & -0.14372700 & 0.55329700 \\ 3.30992800 & 0.58258600 & 1.36887400 \\ 1.88643500 & -0.07012800 & -0.11481800 \\ 0.62095100 & -0.31323000 & 1.20208700 \\ -2.05286100 & -0.75377700 & -0.53380500 \\ -1.66182200 & 0.54557100 & 0.12926400 \\ -4.40881800 & 0.45552900 & 0.06608200 \\ -3.71275300 & -1.28998300 & 0.01916000 \\ -2.05102100 & -0.61750900 & -1.62120400 \\ -3.48992000 & 1.32835900 & -0.09546000 \\ -5.60879100 & 0.59149900 & 0.23572100 \\ -1.33932900 & -1.53369000 & -0.27217400 \\ 3.42394800 & -1.14384000 & 0.96883400 \\ -0.96555100 & 1.62912500 & -0.64603700 \\ -0.44905500 & 2.31609300 & 0.02673600 \\ -0.22819100 & 1.20771800 & -1.33495200 \\ -1.70666900 & 2.18000500 & -1.23456500 \\ 1.74108200 & -1.08407000 & -1.25406200 \\ 2.49558300 & -0.93407300 & -2.03994300 \\ 1.84399100 & -2.10290400 & -0.86355000 \\ 0.75001700 & -1.00403700 & -1.71171700\end{array}$




\section{INT15}

$\mathrm{C}$

$\mathrm{H}$

$\mathrm{H}$

$\mathrm{H}$

$\mathrm{S}$

C

$\mathrm{H}$

C

$\mathrm{S}$

$\mathrm{C}$

C

$\mathrm{H}$

$\mathrm{H}$

$\mathrm{C}$

$\mathrm{H}$

$\mathrm{H}$

H

$\mathrm{H}$

$\mathrm{C}$

$\mathrm{H}$

$\mathrm{H}$

H

$\mathrm{H}$

$\mathrm{H}$

$\mathrm{S}$

INT16

$\mathrm{C}$

$\mathrm{H}$

$\mathrm{H}$

$\mathrm{H}$

$\mathrm{S}$

C

$\mathrm{H}$

C

H

$\mathrm{S}$

C

$\mathrm{O}$

C

$\begin{array}{ccc}-2.56830100 & 1.60107500 & 0.44060200 \\ -2.81857800 & 1.30227700 & 1.46338400 \\ -3.20352100 & 2.44124800 & 0.14543300 \\ -1.51088200 & 1.89703700 & 0.40433000 \\ -2.85534100 & 0.21953000 & -0.73409400 \\ -1.14513800 & -0.46711300 & -0.86518300 \\ -0.50573700 & 0.32069000 & -1.26680600 \\ -0.53494900 & -0.95757600 & 0.44837900 \\ 1.10989900 & -1.75425300 & 0.13434900 \\ 2.35930300 & -0.40344900 & -0.16809600 \\ 2.29131800 & 0.78539100 & 0.82072500 \\ 2.12303000 & 0.36752900 & 1.82286300 \\ -1.22265400 & -1.28569200 & -1.58860800 \\ -1.38371200 & -1.99774200 & 1.17917900 \\ -2.38457200 & -1.59737000 & 1.36955000 \\ -1.49976500 & -2.90883700 & 0.57684500 \\ -0.92087300 & -2.27600400 & 2.13122300 \\ -0.34514500 & -0.08584300 & 1.08098200 \\ 2.43073700 & 0.02541400 & -1.63219100 \\ 2.48008600 & -0.84202600 & -2.30021300 \\ 1.56910800 & 0.64812000 & -1.88098800 \\ 3.32622300 & 0.64370400 & -1.78568100 \\ 3.27403800 & -0.97051400 & 0.06697700 \\ 3.31023200 & 1.20665000 & 0.83396300 \\ 1.09753300 & 2.13616200 & 0.44888500\end{array}$

$-2.80728800$

2.40311500

0.47440800

$-3.45169000$

3.02991500

1.09668700

$-2.29996100$

3.02774400

$-0.26636700$

$-2.05304500$

1.91414500

1.09408600

$-3.86678700$

1.15485800

$-0.34860500$

$-2.55151600$

$-0.03399900$

$-0.85193500$

$-1.68864700$

0.55095000

$-1.17344000$

$-2.15673300$

$-1.00587800$

0.26564300

$-1.96267100$

$-0.43487200$

1.17637400

$-0.55669900$

$-1.81834600$

$-0.14973000$

0.55878700

$-0.37851500$

0.25992800

0.08914800

0.62993800

0.75901100

3.01970500

0.80358700 


$\begin{array}{lrrr}\mathrm{H} & 2.56191400 & 1.03240500 & 1.44210100 \\ \mathrm{C} & 2.84248500 & 1.95489100 & -0.51199600 \\ \mathrm{H} & 1.78842800 & 2.16185800 & -0.72392800 \\ \mathrm{H} & 3.29548900 & 2.86715600 & -0.09843800 \\ \mathrm{H} & 3.36594200 & 1.68765400 & -1.43526600 \\ \mathrm{C} & 4.48285400 & 0.28061900 & 0.51078700 \\ \mathrm{H} & 4.59206500 & -0.26002500 & 1.49975800 \\ \mathrm{~S} & 2.22308700 & -0.73605000 & -0.22018000 \\ \mathrm{H} & -2.93221000 & -0.58346000 & -1.71899900 \\ \mathrm{C} & -3.22210300 & -2.06837000 & 0.52430000 \\ \mathrm{H} & -4.17552700 & -1.58253000 & 0.76182300 \\ \mathrm{H} & -3.37173800 & -2.70200600 & -0.35842700 \\ \mathrm{H} & -2.93894000 & -2.71336700 & 1.36161800 \\ \mathrm{H} & 5.13027200 & 1.20247000 & 0.63536000 \\ \mathrm{O} & 4.70454500 & -0.44720200 & -0.59010500\end{array}$



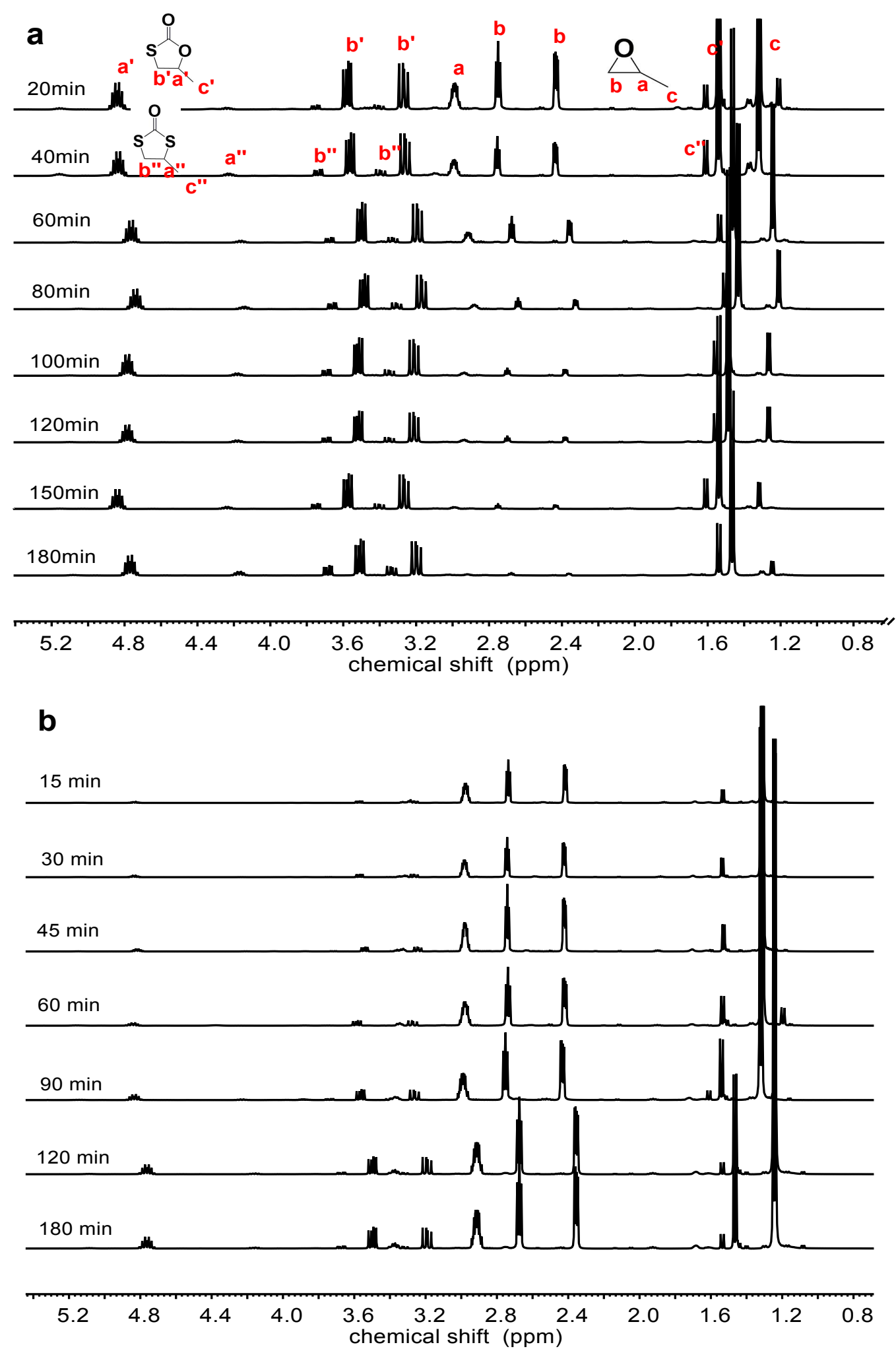

Figure S8. ${ }^{1} \mathrm{H}$ NMR of the crude products varied with time at $80^{\circ} \mathrm{C}$ with a) $\mathrm{PO} / \mathrm{COS} /$ $\mathrm{DBU} / \mathrm{TU}=100 / 1.2 / 1 / 2$, b) $\mathrm{PO} / \mathrm{COS} / \mathrm{DBU}=100 / 1.2 / 1.0$. 

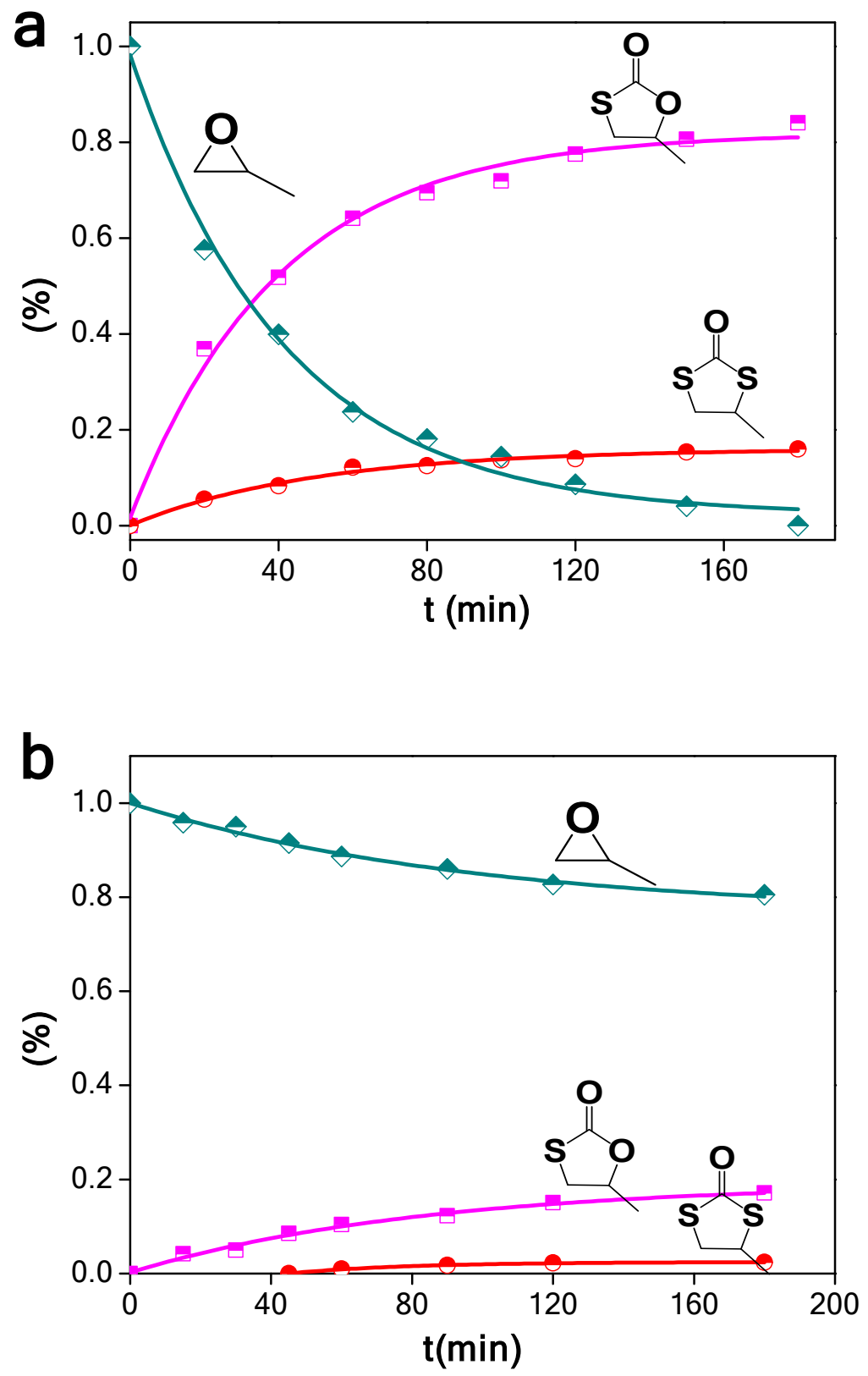

Figure S9. The content of multiple components varied with time at $80^{\circ} \mathrm{C}$ with a) DBU/TU, b) single DBU. 


\section{a}

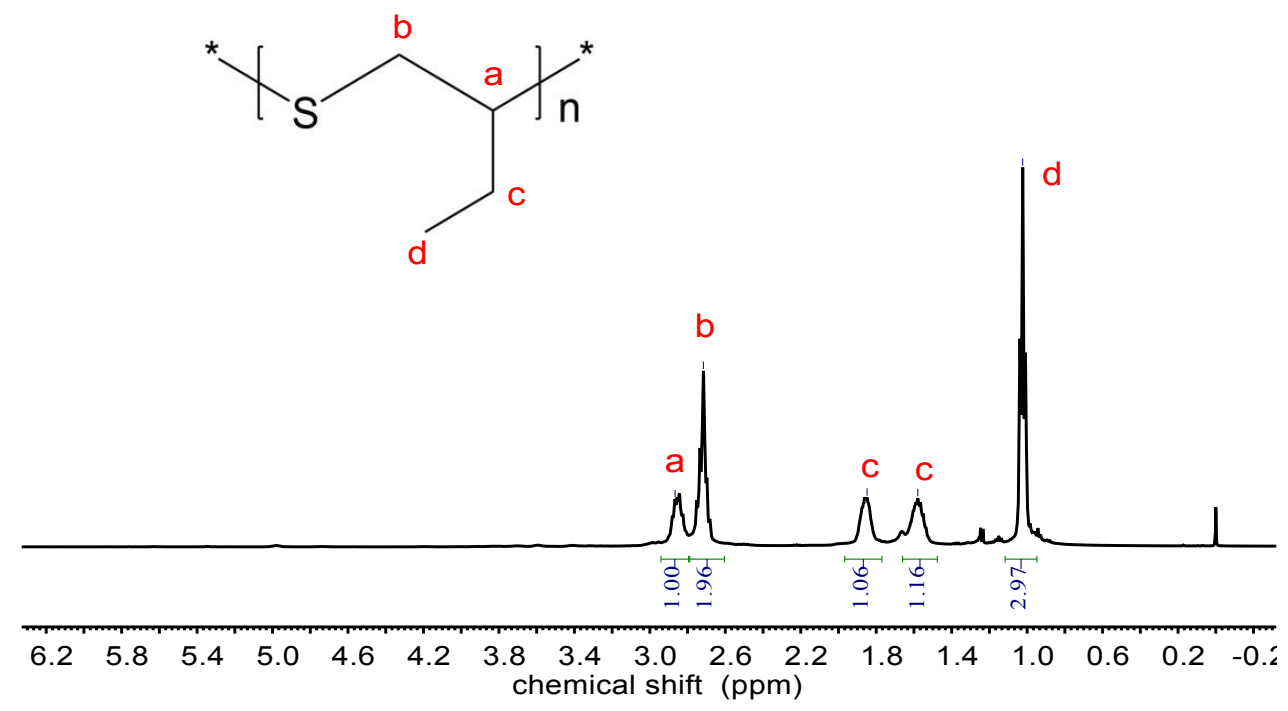

b

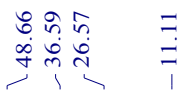

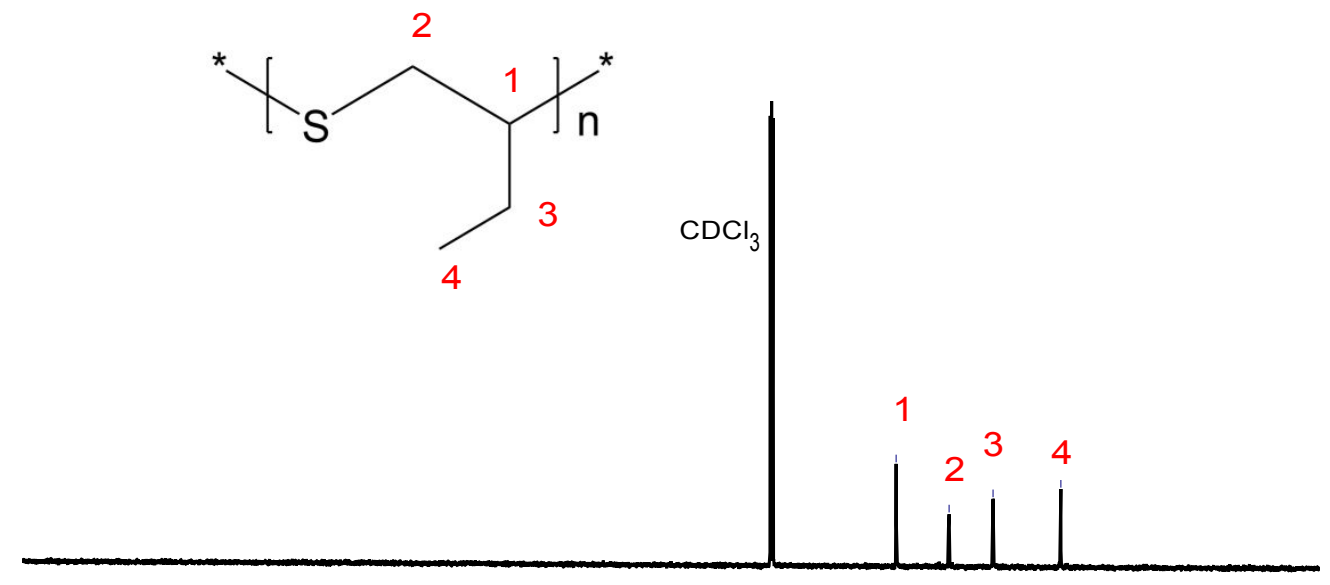

\begin{tabular}{lllllllllllllll}
\hline 240 & 220 & 200 & 180 & 160 & 140 & 120 & 100 & 80 & 60 & 40 & 20 & 0 & -20 & -40 \\
chemical shift & $(\mathrm{ppm})$
\end{tabular}

Figure S10. (a) ${ }^{1} \mathrm{H}$ NMR and (b) ${ }^{13} \mathrm{C}$ NMR of the generated poly(thioether) from epoxide 2, Table 3. 
a

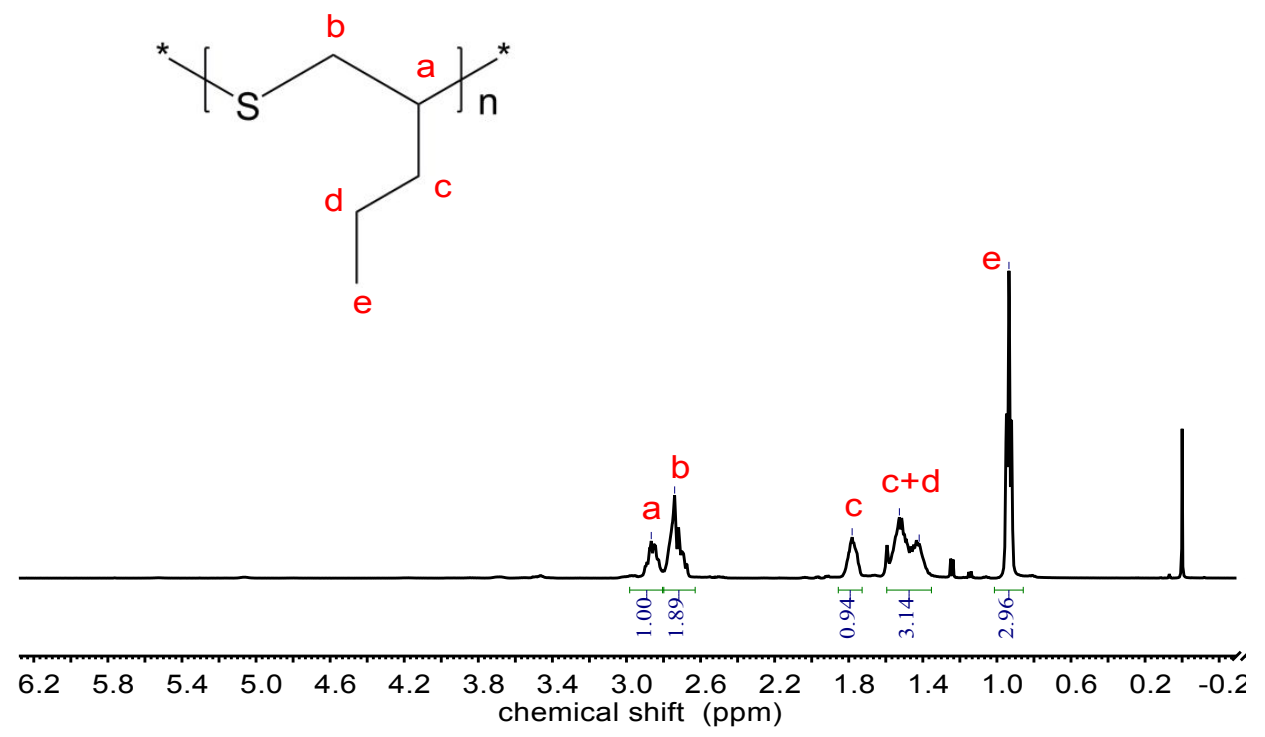

b

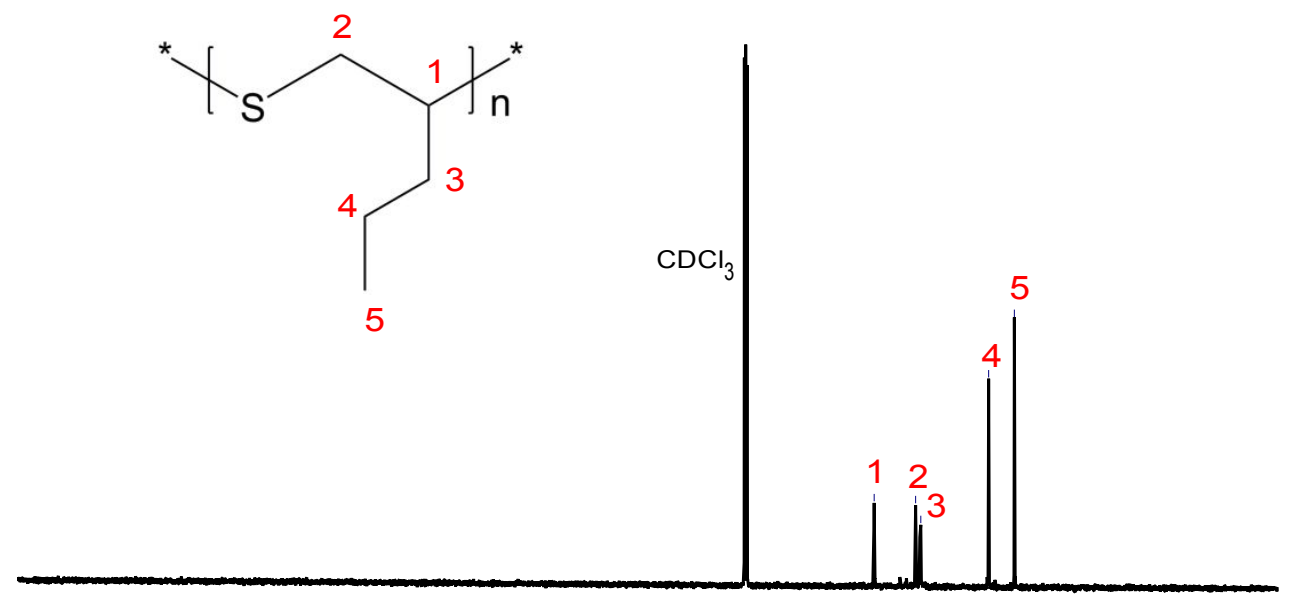

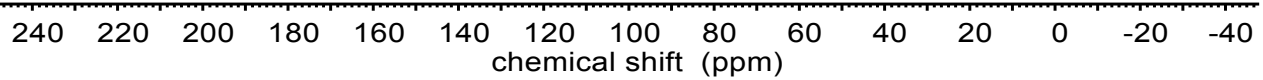

Figure S11. (a) ${ }^{1} \mathrm{H}$ NMR and (b) ${ }^{13} \mathrm{C}$ NMR of the generated poly(thioether) from epoxide 3, Table 3. 
a

$\underset{\substack{\infty \\ i}}{1}$

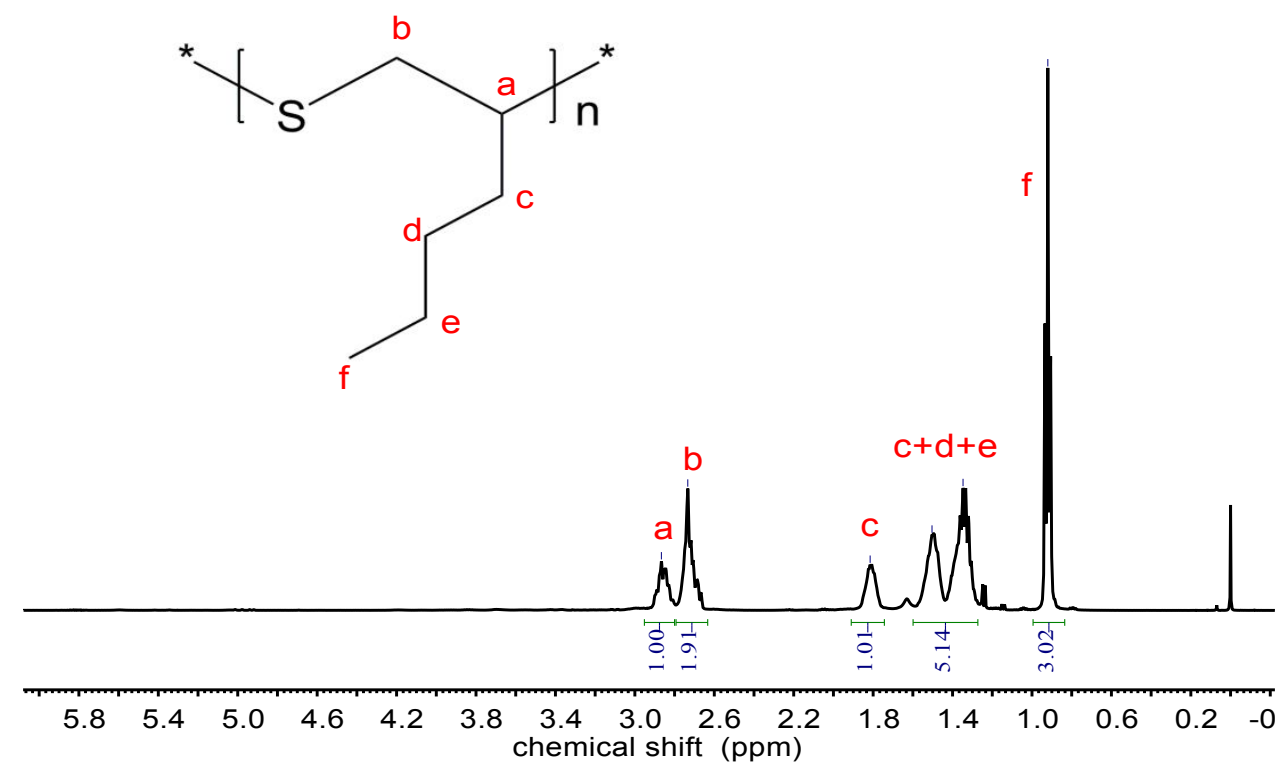

b

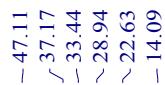

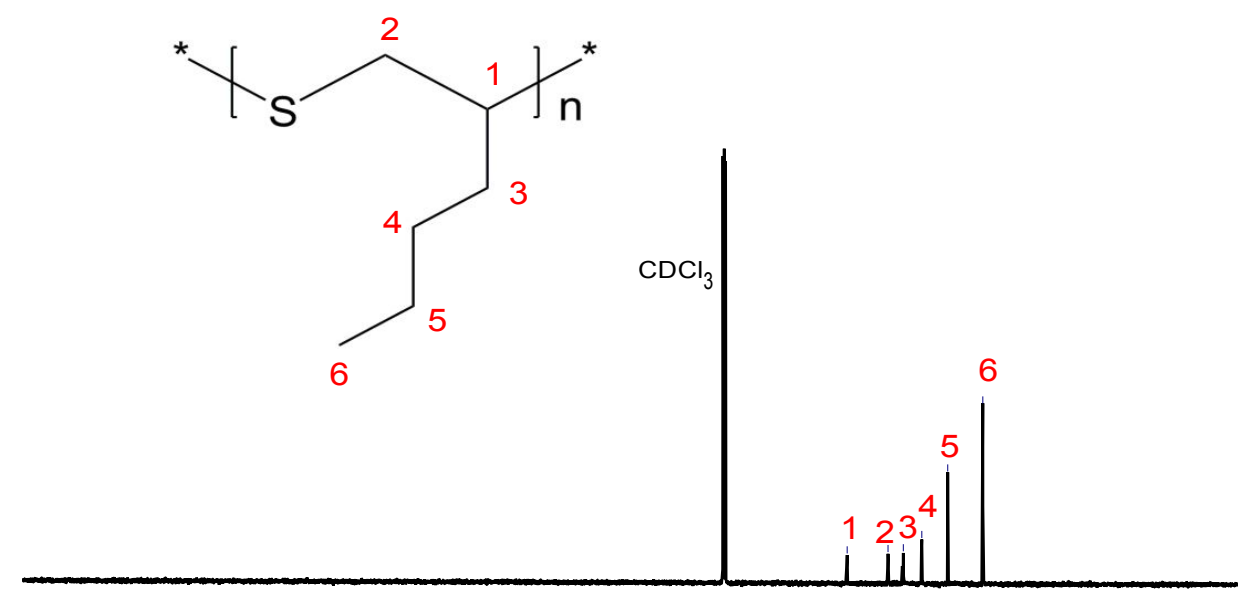

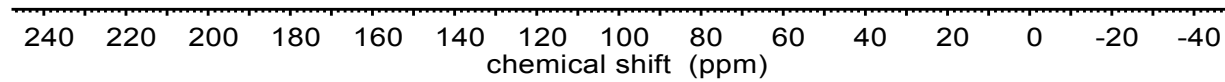

Figure S12. (a) ${ }^{1} \mathrm{H}$ NMR and (b) ${ }^{13} \mathrm{C}$ NMR of the generated poly(thioether) from epoxide 4, Table 3. 


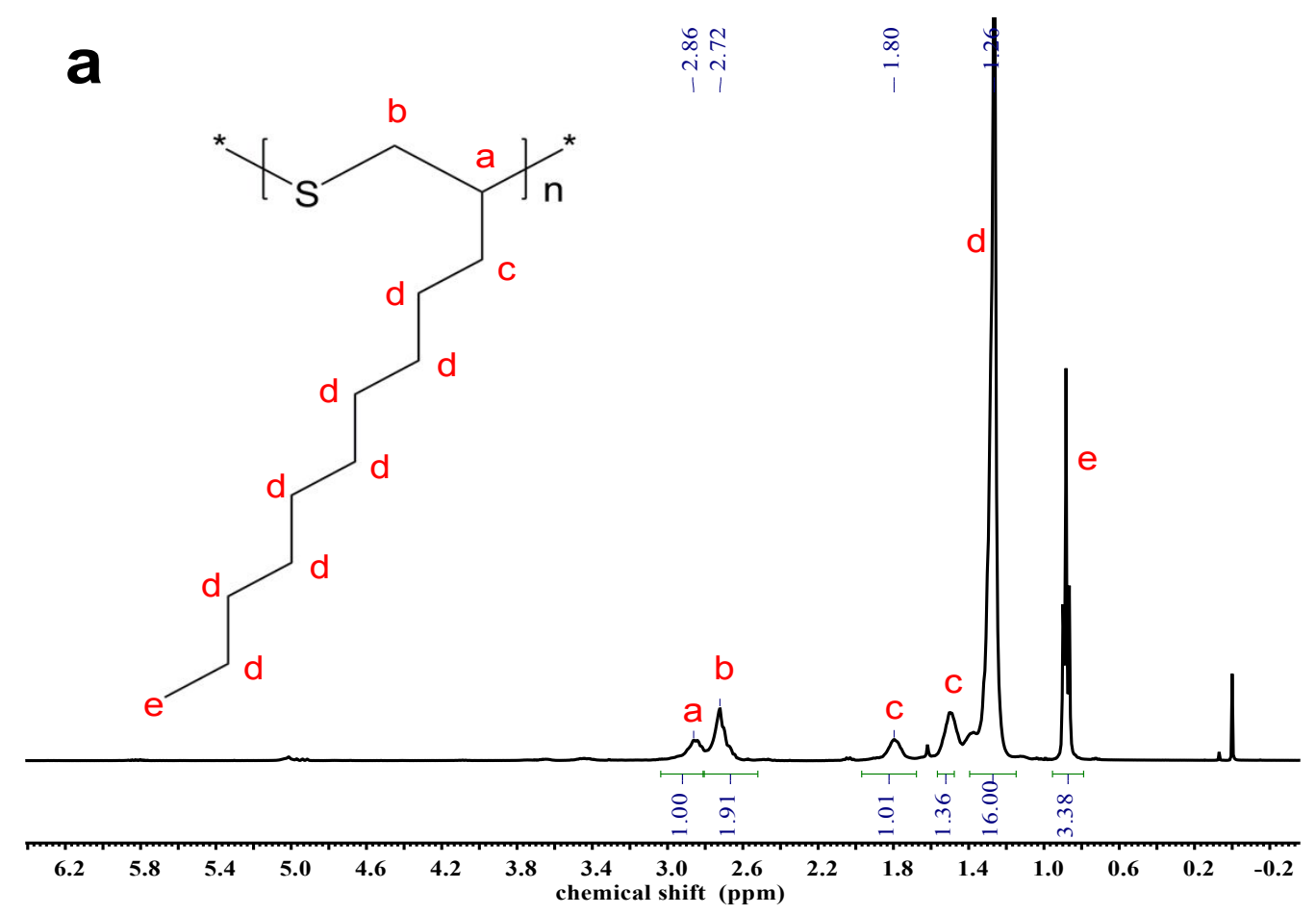

b
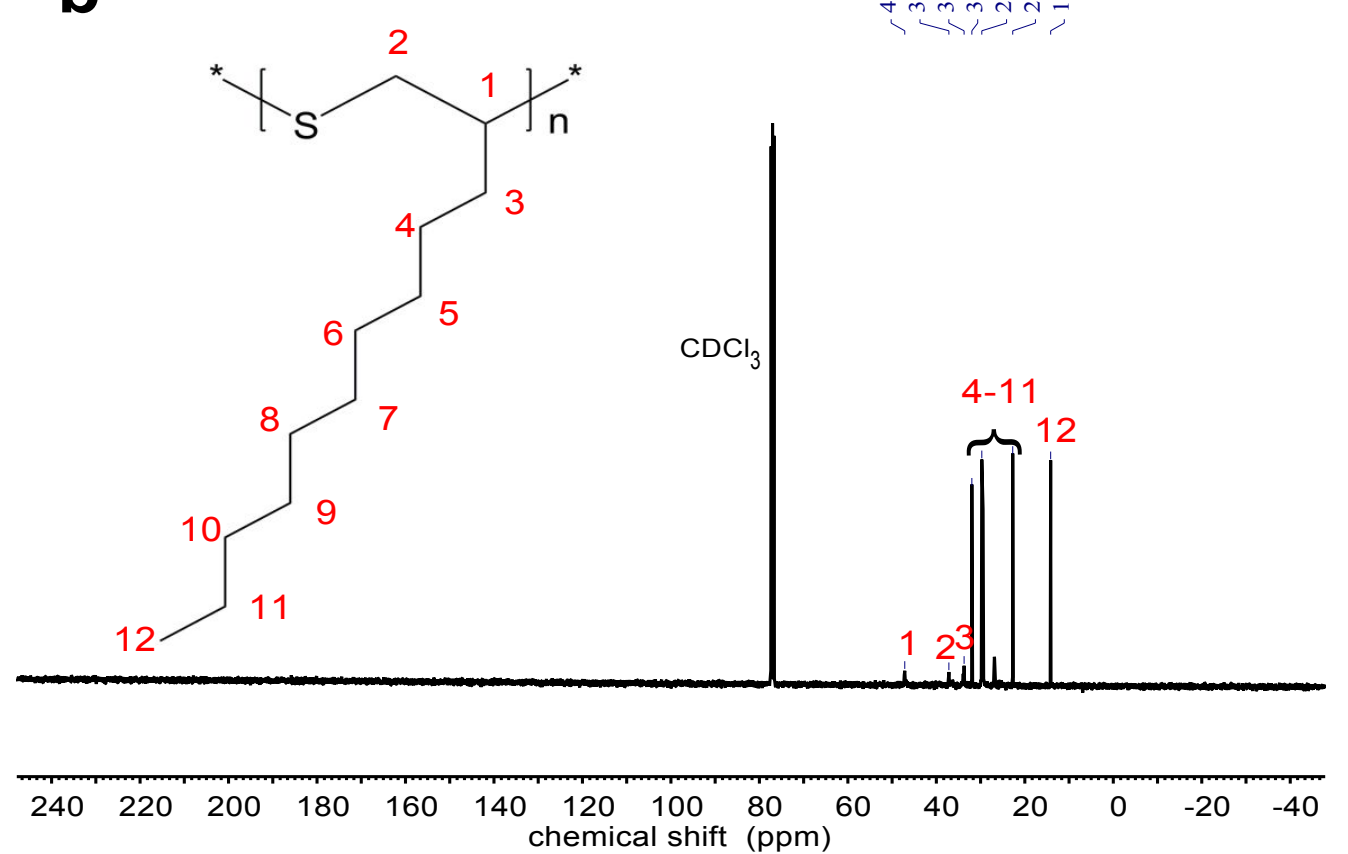

Figure S13. (a) ${ }^{1} \mathrm{H}$ NMR and (b) ${ }^{13} \mathrm{C}$ NMR of the generated poly(thioether) from epoxide 8, Table 3. 

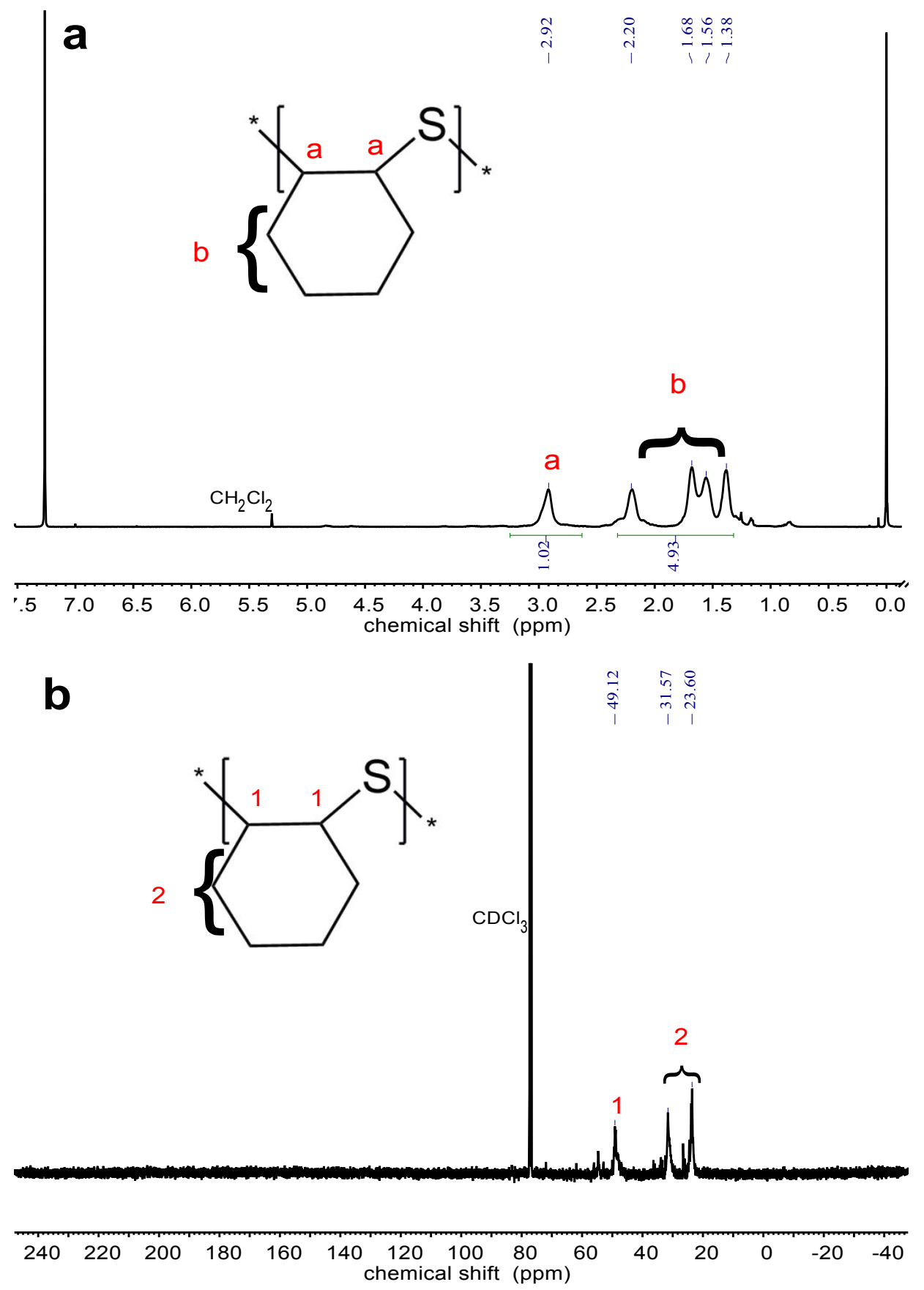

Figure S14. (a) ${ }^{1} \mathrm{H}$ NMR and (b) ${ }^{13} \mathrm{C}$ NMR of the generated poly(thioether) from epoxide 9, Table 3. 


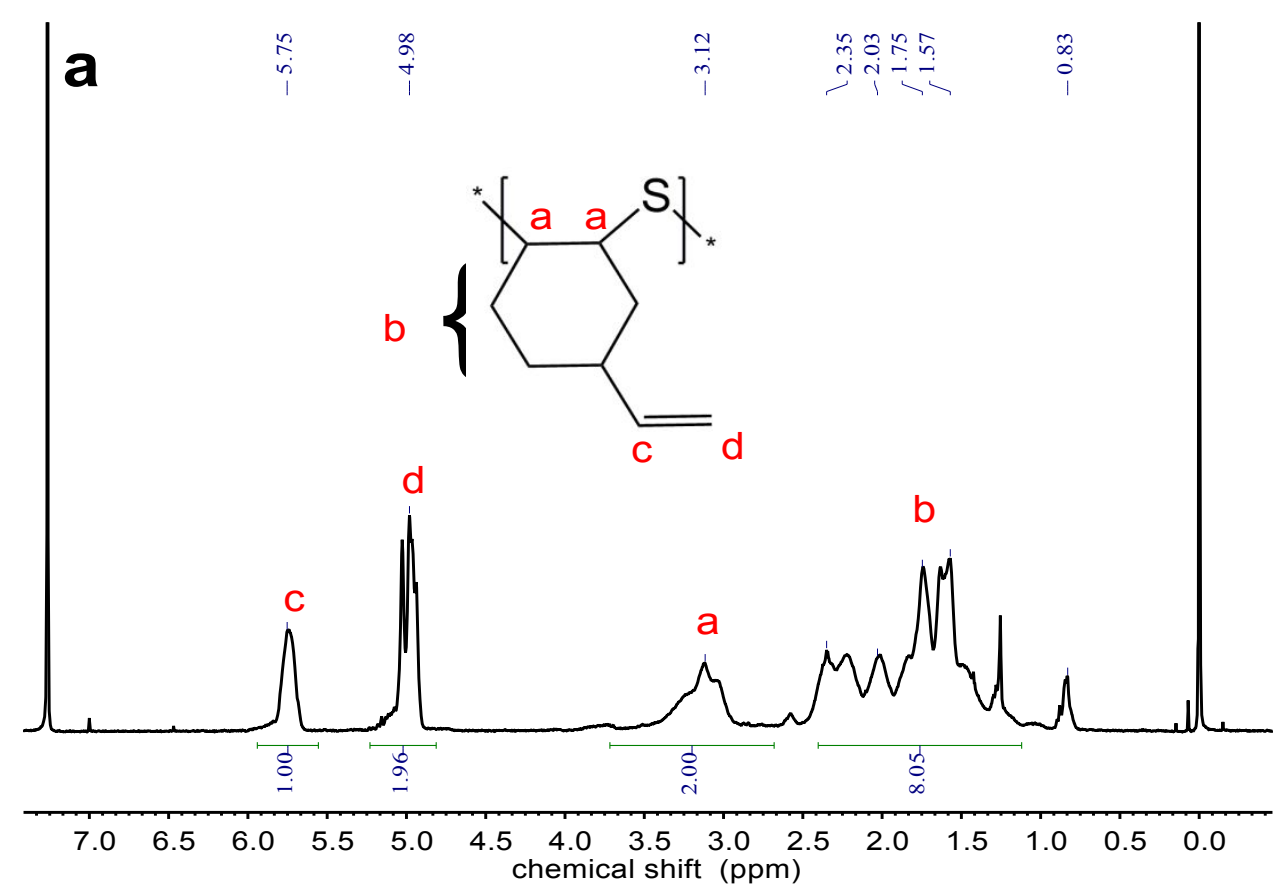

b
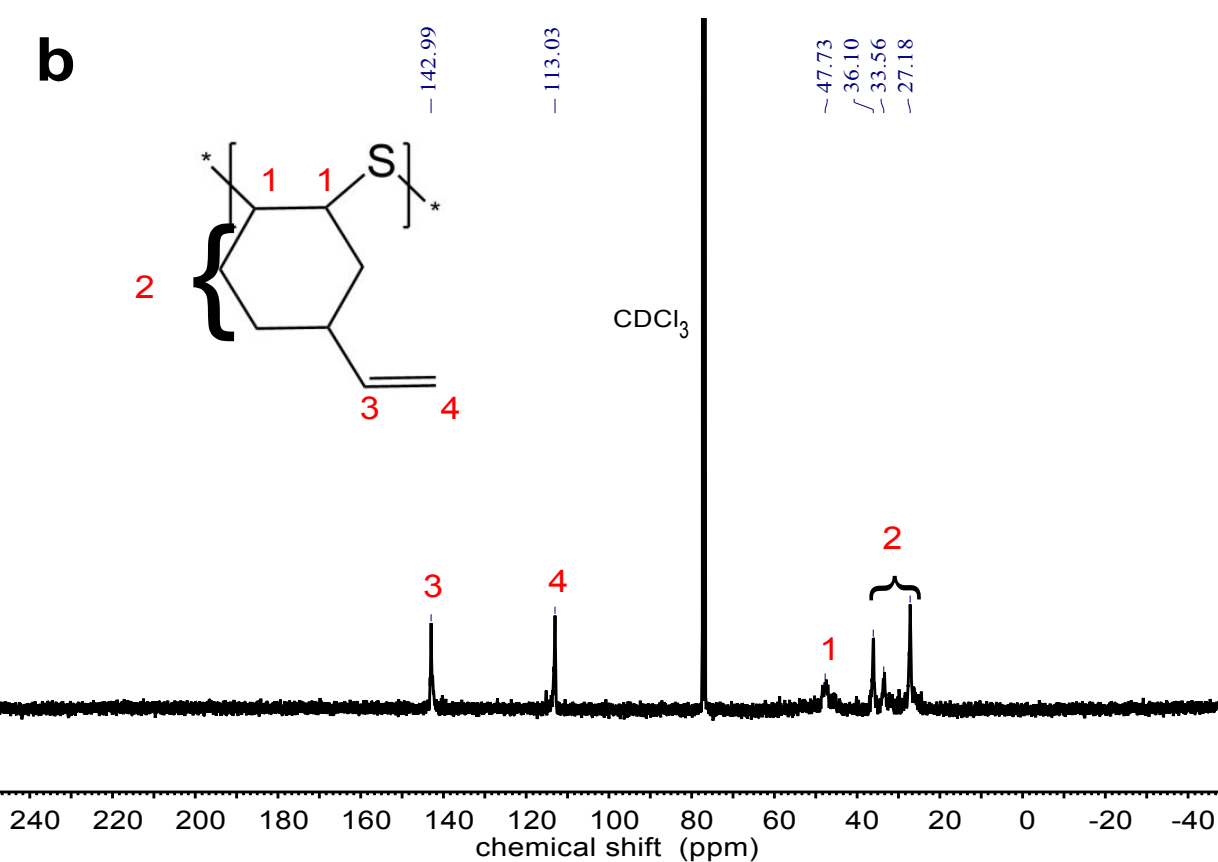

Figure S15. (a) ${ }^{1} \mathrm{H}$ NMR and (b) ${ }^{13} \mathrm{C}$ NMR of the generated poly(thioether) from epoxide 10, Table 3. 

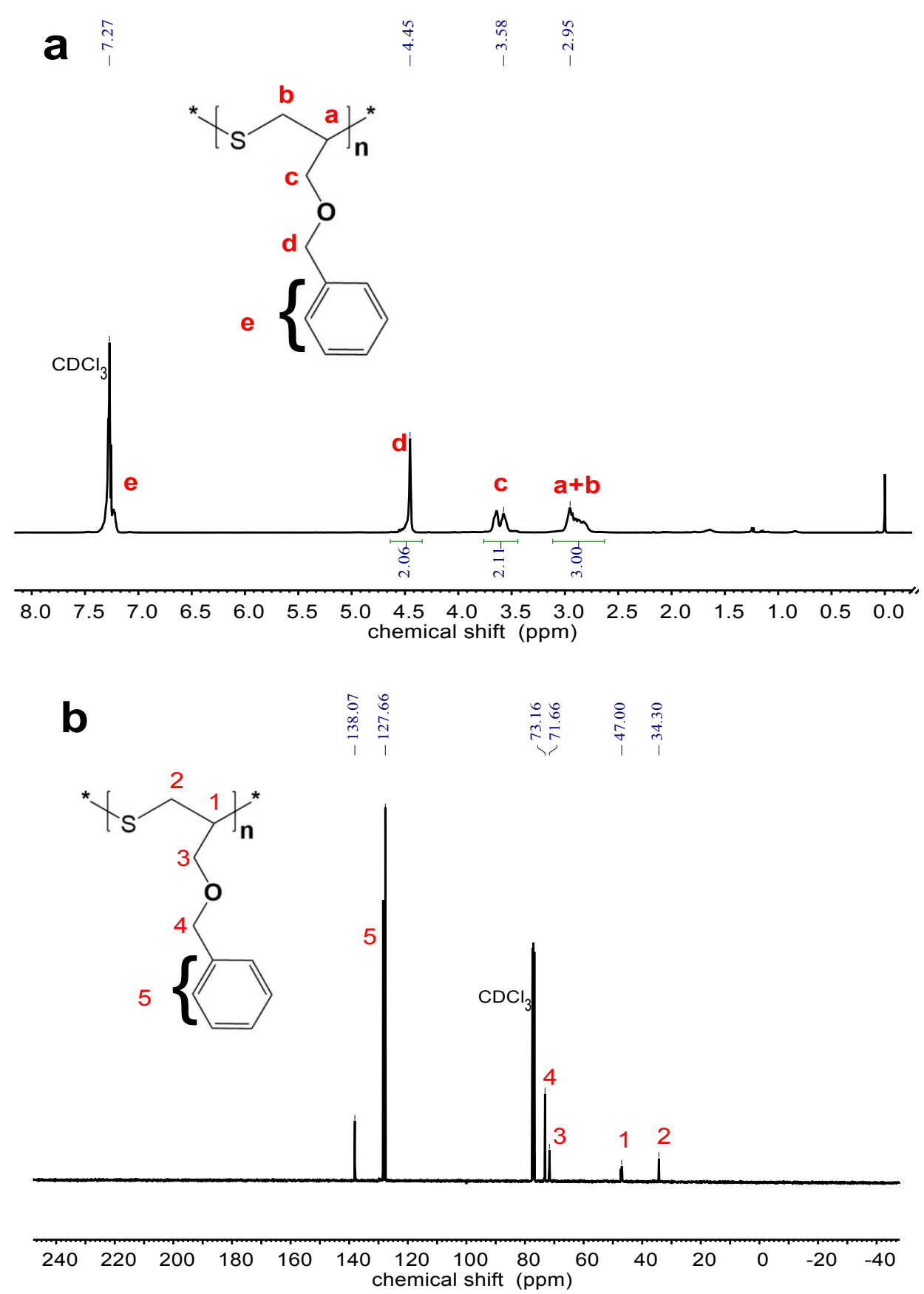

Figure S16. (a) ${ }^{1} \mathrm{H}$ NMR and (b) ${ }^{13} \mathrm{C}$ NMR of the generated poly(thioether) from epoxide 11, Table 3. 

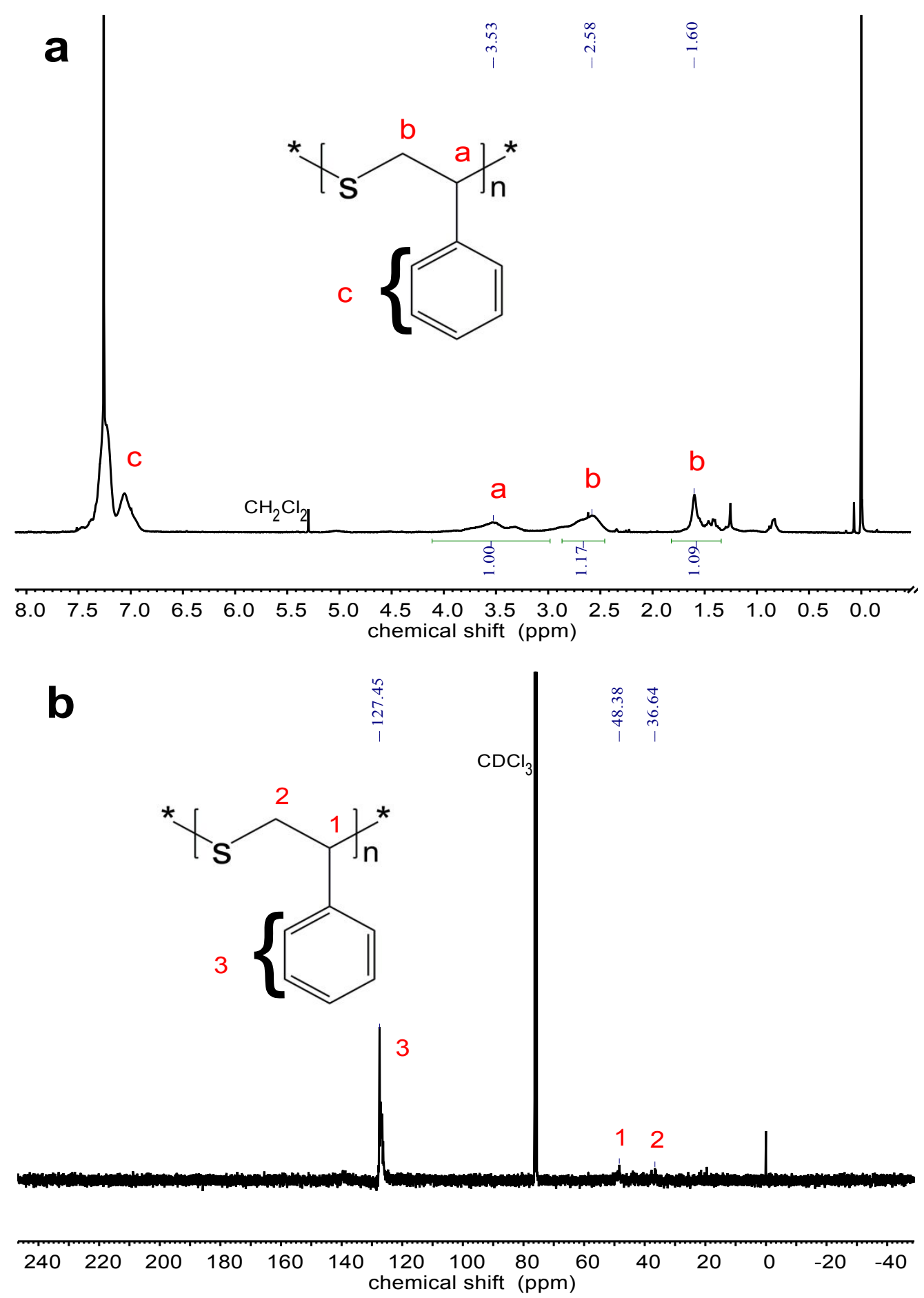

Figure S17. (a) ${ }^{1} \mathrm{H}$ NMR and (b) ${ }^{13} \mathrm{C}$ NMR of the generated poly(thioether) from epoxide 12, Table 3. 

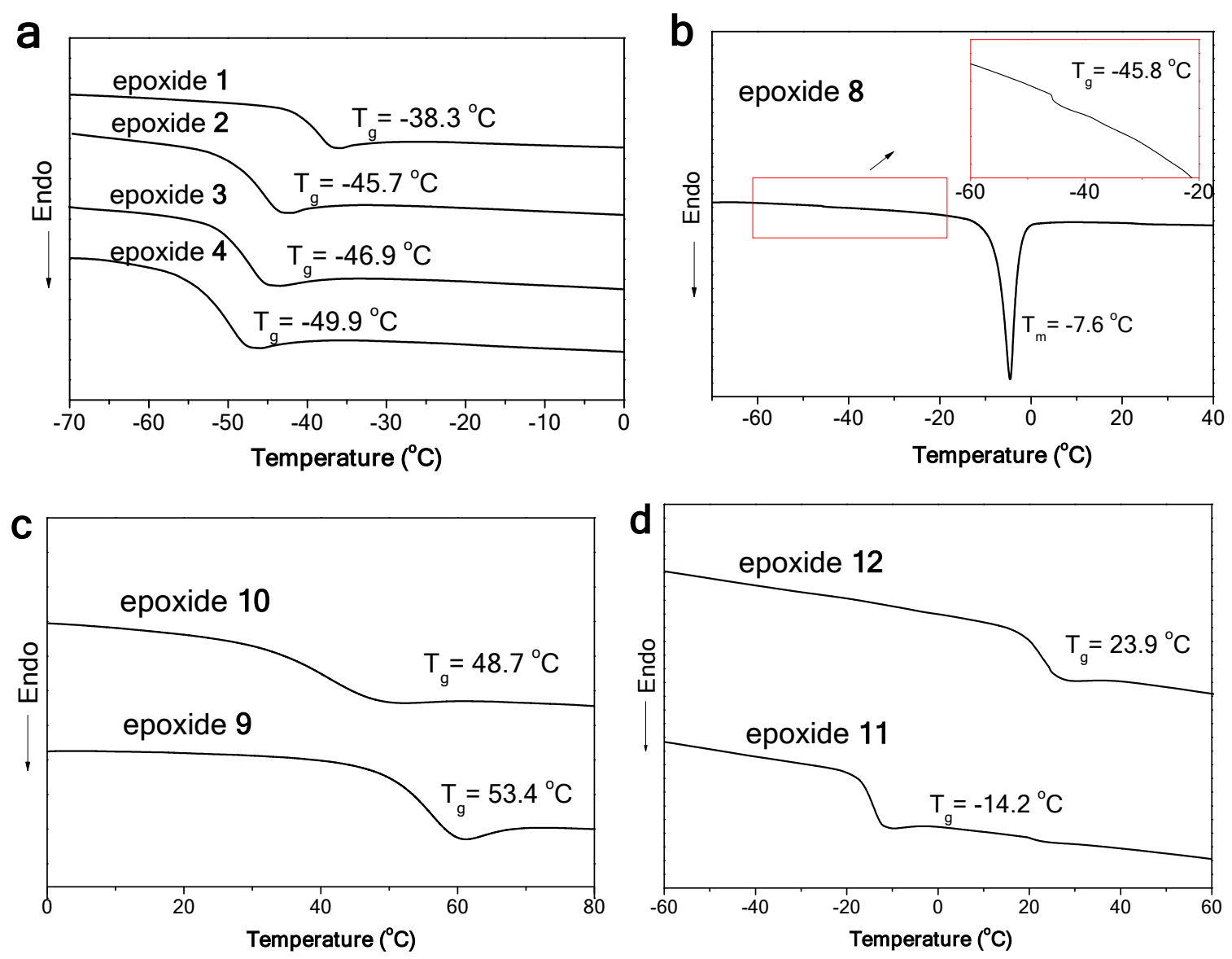

Figure S18. DSC curves of the generated poly(thioether)s from a) epoxides 1-4, b) epoxide 8, c) epoxides 9-10 and d) epoxides 11-12 in Table 3. 


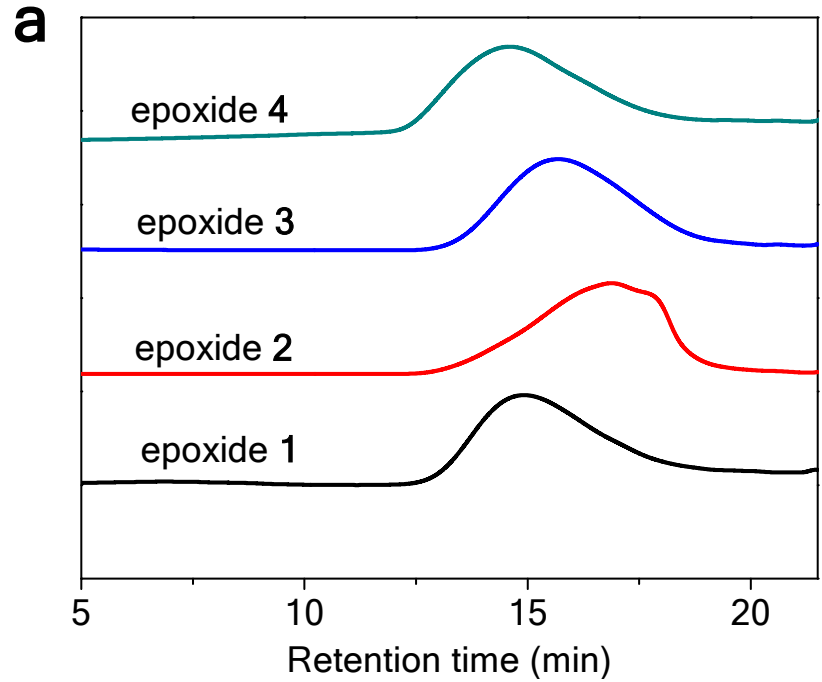

b
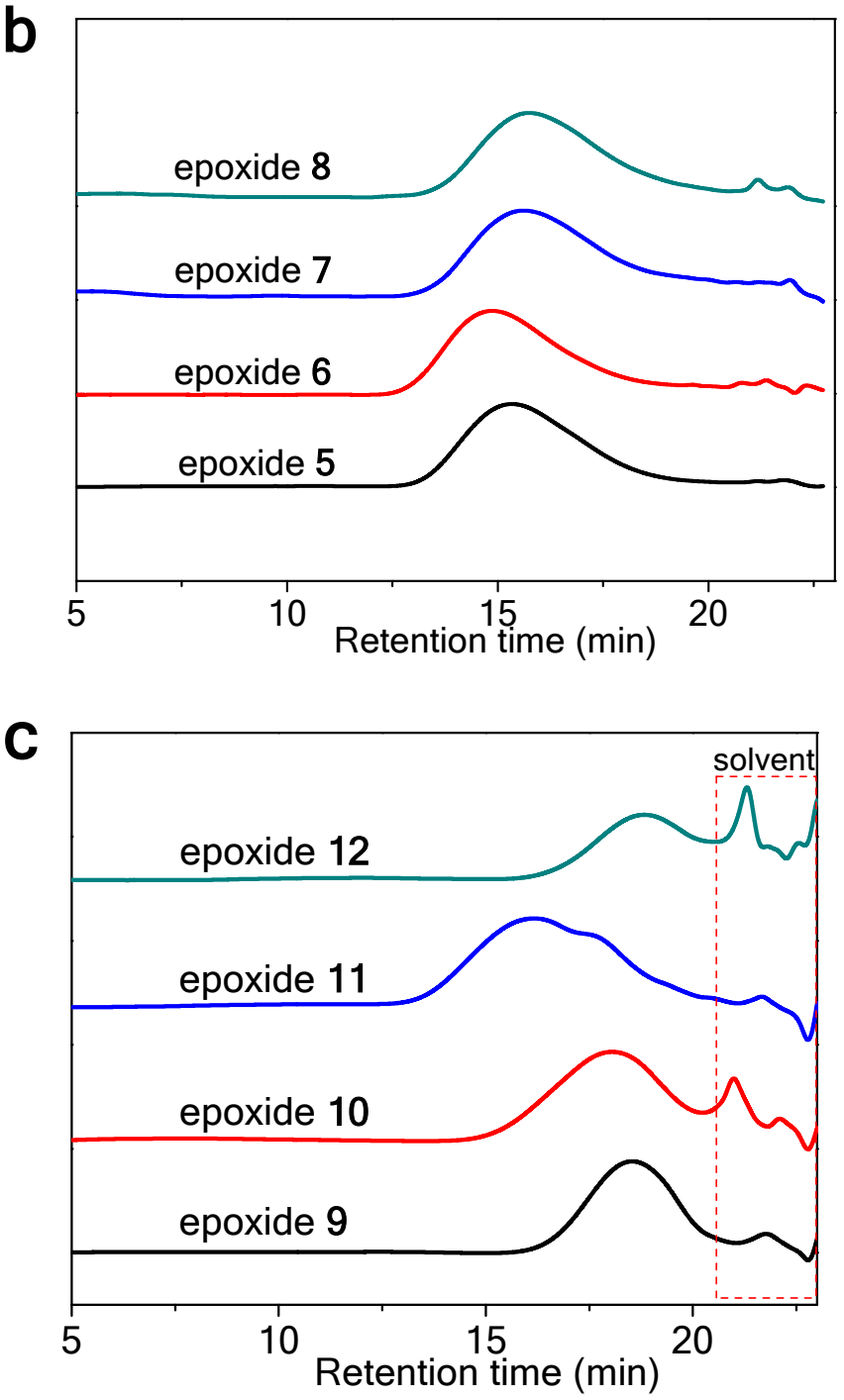

Figure S19. GPC curves of the generated poly(thioether)s from a) epoxides 1-4, b) epoxides 5-8, c) epoxides 9-12 in Table 3. 


\section{Reference}

1. Frisch, M. J. T., G. W.; Schlegel, H. B.; Scuseria, G. E.;; Robb, M. A. C., J. R.; Scalmani, G.; Barone, V.; Mennucci,; B.; Petersson, G. A. N., H.; Caricato, M.; Li, X.; Hratchian, H.; P.; Izmaylov, A. F. B., J.; Zheng, G.; Sonnenberg, J. L.; Hada, M.;; Ehara, M. T., K.; Fukuda, R.; Hasegawa, J.; Ishida, M.; Nakajima,; T.; Honda, Y. K., O.; Nakai, H.; Vreven, T.; Montgomery, J. A., Jr.;; Peralta, J. E. O., F.; Bearpark, M.; Heyd, J. J.; Brothers, E.; Kudin,; K. N.; Staroverov, V. N. K., R.; Normand, J.; Raghavachari, K.;; Rendell, A. B., J. C.; Iyengar, S. S.; Tomasi, J.; Cossi, M.; Rega,; N.; Millam, J. M. K., M.; Knox, J. E.; Cross, J. B.; Bakken, V.;; Adamo, C. J., J.; Gomperts, R.; Stratmann, R. E.; Yazyev, O.;; Austin, A. J. C., R.; Pomelli, C.; Ochterski, J. W.; Martin, R. L.;; Morokuma, K. Z., V. G.; Voth, G. A.; Salvador, P.;; Dannenberg, J. J. D., S.; Daniels, A. D.; Farkas, O.;; Foresman, J. B. O., J. V.; Cioslowski, J.; Fox, D. J.; , Gaussian 09, revision C.01; . Gaussian Inc.: Wallingford, CT, 2010.

2. Becke, A. D., Density-functional thermochemistry. III. The role of exact exchange. J. Chem. Phys. 1993, 98 (7), 5648-5652.

3. Lee, C.; Yang, W.; Parr, R. G., Development of the Colle-Salvetti correlation-energy formula into a functional of the electron density. Physical Review B 1988, 37 (2), 785-789.

4. Grimme, S.; Antony, J.; Ehrlich, S.; Krieg, H., A consistent and accurate ab initio parametrization of density functional dispersion correction (DFT-D) for the 94 elements $\mathrm{H}-\mathrm{Pu} . J$. Chem. Phys. 2010, 132 (15), 154104.

5. Tomasi, J.; Mennucci, B.; Cammi, R., Quantum mechanical continuum solvation models. Chem. Rev. 2005, 105 (8), 2999-3093.

6. Cancès, E.; Mennucci, B., Comment on "Reaction field treatment of charge penetration" [J. Chem. Phys. 112, 5558 (2000)]. J. Chem. Phys. 2001, 114 (10).

7. Chipman, D. M., Reaction field treatment of charge penetration. J. Chem. Phys. 2000, 112 (13), 5558-5565.

8. Legault, C. Y., CYLView. CYLView 2009, 1.0b, http://www.cylview.org 